\title{
THE
}

10-1-1976

\section{Spin-Peierls transitions in magnetic donor-acceptor compounds of tetrathiafulvalene (TTF) with bisdithiolene metal complexes}

\author{
I. S. Jacobs \\ J. W. Bray \\ H. R. Hart Jr. \\ L. V. Interrante \\ J. S. Kasper
}

See next page for additional authors

Follow this and additional works at: https://digitalcommons.uri.edu/phys_facpubs

Terms of Use

All rights reserved under copyright.

\section{Citation/Publisher Attribution}

Jacobs, I. S., Bray, J. W., Hart, H. R., Interrrante, L. V., Kasper, J. S., Watkins, G. D., Prober, D. E., \& Bonner, J. C. (1976). Phys. Rev. B, 14(7), 3036-3051. doi: 10.1103/PhysRevB.14.3036

Available at: http://dx.doi.org/10.1103/PhysRevB.14.3036

This Article is brought to you for free and open access by the Physics at DigitalCommons@URI. It has been accepted for inclusion in Physics Faculty Publications by an authorized administrator of DigitalCommons@URI. For more information, please contact digitalcommons-group@uri.edu. 


\section{Authors}

I. S. Jacobs, J. W. Bray, H. R. Hart Jr., L. V. Interrante, J. S. Kasper, G. D. Watkins, D. E. Prober, and Jill C. Bonner

This article is available at DigitalCommons@URI: https://digitalcommons.uri.edu/phys_facpubs/130 


\title{
Spin-Peierls transitions in magnetic donor-acceptor compounds of tetrathiafulvalene (TTF) with bisdithiolene metal complexes*
}

\author{
I. S. Jacobs, J. W. Bray, H. R. Hart, Jr., L. V. Interrante, J. S. Kasper, and G. D. Watkins ${ }^{\dagger}$ \\ General Electric Company, Corporate Research and Development, Schenectady, New York 12301 \\ D. E. Prober \\ Department of Engineering and Applied Science, Yale University, New Haven, Connecticut 06520 \\ J. C. Bonner \\ Applied Mathematics Department, Brookhaven National Laboratory, Upton, New York 11973 \\ (Received 12 May 1976)
}

\begin{abstract}
The spin-Peierls transition is considered as a progressive spin-lattice dimerization occurring below a transition temperature in a system of one-dimensional antiferromagnetic Heisenberg chains. In the simplest theories, the transition is second order and the ground state is a singlet with a magnetic gap. The historical origins and theoretical development of the concept are examined. Magnetic susceptibility and EPR measurements on the $\pi$ donor-acceptor compounds TTF. $M S_{4} \mathrm{C}_{4}\left(\mathrm{CF}_{3}\right)_{4}(M=\mathrm{Cu}, \mathrm{Au}$; TTF is tetrathiafulvalene) are reported. These compounds exhibit clearly the characteristics of the spin-Peierls transition in reasonably good agreement with a mean-field theory. The susceptibility of each compound has a broad maximum near $50 \mathrm{~K}$, while the transitions occur at 12 and $2.1 \mathrm{~K}$ for $M=\mathrm{Cu}$ and $\mathrm{Au}$, respectively. EPR linewidth observations over a broad temperature range are examined. Areas for further experimental and theoretical work are indicated, and a critical comparison is made of related observations on other materials.
\end{abstract}

\section{INTRODUCTION}

We have recently reported ${ }^{1}$ evidence for a spinPeierls transition, i.e., a progressive spin-lattice dimerization occurring below a transition temperature in a system of one-dimensional $(1-D)$ antiferromagnetic (AF) Heisenberg chains. The transition is second order and is driven by the spin subsystem. At the lowest temperature the system is in a singlet ground state with a magnetic gap.

The description of this spin-lattice effect combines two active areas of solid-state science with potentially fruitful consequences for the further development of each. One of these areas is the rich one of metal-nonmetal transitions. ${ }^{2}$ The major feature of interest to us here is the family of lattice distortion models typified by the Peierls instability. ${ }^{3}$ The second area contains magnetic model systems, ${ }^{4}$ usually insulators, including those of less than three dimensions. The combination of exact solutions, approximation techniques and experimental comparisons in this area has contributed importantly to the knowledge of phase transitions and critical phenomena. Our focus herein is on one-dimensional $(1-D)$ magnetic models embedded in a $3-\mathrm{D}$ lattice.

The specific concept of the spin-Peierls transition, i.e., the instability of one-dimensional spininsulator systems, has seen theoretical development for over a decade, as we describe in Sec. II. Several materials have been put forth as test examples during this time. In most cases, the spin-
Peierls nature has been disputed after closer study. We review these in Sec. V, after presenting our experiments and results in Sec. III and analyzing the latter in Sec. IV.

We have carried out our experiments on several tetrathiafulvalene donor-acceptor compounds with bisdithiolene metal complexes..$^{1,5,6}$ The planar organic molecular units of these compounds put them into the broader class of molecular crystals ${ }^{7}$ which have been attracting increasing attention from solid-state scientists after having been predominantly in the chemist's domain.

\section{HISTORICAL REVIEW}

In their studies of the paramagnetic resonance of solid free radicals, McConnell and co-workers ${ }^{8}$ seem to have been the first (in 1962) to apply the instability against dimerization of a linear system to magnetic chains of spin $\frac{1}{2}$. They drew upon various background sources, particularly citing the example worked by Peierls ${ }^{3}$ showing that a onedimensional metallic lattice of uniformly spaced atoms with a half-filled conduction band prefers to become a distorted lattice in which alternate atoms are displaced in opposite directions. The resulting gap at the Fermi surface implies that the material becomes a semiconductor (nonmetal). Concurrently, Frölich ${ }^{9}$ and Kuper ${ }^{10}$ treated such a model because of its possible relevance to superconductivity. A second background source cited was the study of the alternation of bond lengths in long conjugated chain molecules, the preference 
for alternation developing if the chain is sufficiently long. The works of Ooshika ${ }^{11}$ and of LonguetHiggins and Sale ${ }^{12}$ are to be noted in this regard, with credit for seminal ideas traced back to Platt ${ }^{13}$ and earlier work by Kuhn. ${ }^{14}$

McConnell et $a l .^{8}$ qualitatively predicted a transition at temperatures comparable to or less than the intrachain exchange coupling, including a "sharp" decrease in the magnetic susceptibility. The first quantitative treatment, to our knowledge, was that of Chesnut. ${ }^{15}$ He chose a model with a familiar spin-spin coupling Hamiltonian, a separation-dependent exchange expanded to the linear term, and an elastic lattice. Mean-field calculations with Heisenberg and Ising interactions result in second-order phase transitions from a uniformly spaced lattice to one of alternating character. Essentially this behavior is retained in the calculations which followed. Both Chesnut and McConnell et al. were attempting to understand the properties of Würster's blue perchlorate. Despite attractive similarities, the model was judged inadequate. (See Sec. V.)

Along with these developments, there was considerable progress in the description of low-dimensional magnetic systems. We focus on the magnetic susceptibility and low-lying excited states of antifer romagnetic 1 -D Heisenberg $\left(S=\frac{1}{2}\right)$ chains. For uniform exchange between spins along the chain, we cite the studies by Bonner and Fisher ${ }^{16}$ and by Bulaevskii, ${ }^{17}$ which contain many earlier references. Of equal importance to us is the case of nonuniform or alternating exchange along the chain, considered by Bulaevskii, ${ }^{18}$ by Duffy and Barr, ${ }^{19}$ and others cited therein. The spin coupling Hamiltonian for these cases is

$$
H=\sum_{i=1}^{N / 2}\left(J_{1} \overrightarrow{\mathrm{S}}_{2 i} \cdot \overrightarrow{\mathrm{S}}_{2 i-1}+J_{2} \overrightarrow{\mathrm{S}}_{2 i} \cdot \overrightarrow{\mathrm{S}}_{2 i+1}\right)
$$

Henceforth, we define $\alpha \equiv J_{2} / J_{1}$ so that $\alpha=1$ is the uniform chain and $\alpha<1$ is the alternating chain, reaching the limiting case of isolated AF dimers at $\alpha=0$. In Fig. 1 is shown the normalized magnetic susceptibility $\chi_{r}=\chi J_{1} / N g^{2} \mu_{B}^{2}$ vs $k T / J_{1}$ for the cases $\alpha=1,0.95$, and 0 (with $N \rightarrow \infty$ ). The curves are calculated in the Hartree-Fock approximation of Bulaevskii. (The Bonner-Fisher calculation for $\alpha=1$ is more reliable, but the equivalent calculation for $\alpha \lesssim 1$ has not been done. The Bulaevskii curve reproduces the general features of the Bonner-Fisher curve.) The curve for $\alpha=0$ is the familiar singlet-triplet model with $\chi_{r}$ $=\left(J_{1} / k T\right) /\left[\exp \left(J_{1} / k T\right)+3\right]$. The purpose of the figure is to show that the susceptibility for the uniform infinite chain is nonvanishing at absolute zero and that the two nonuniform chains at either

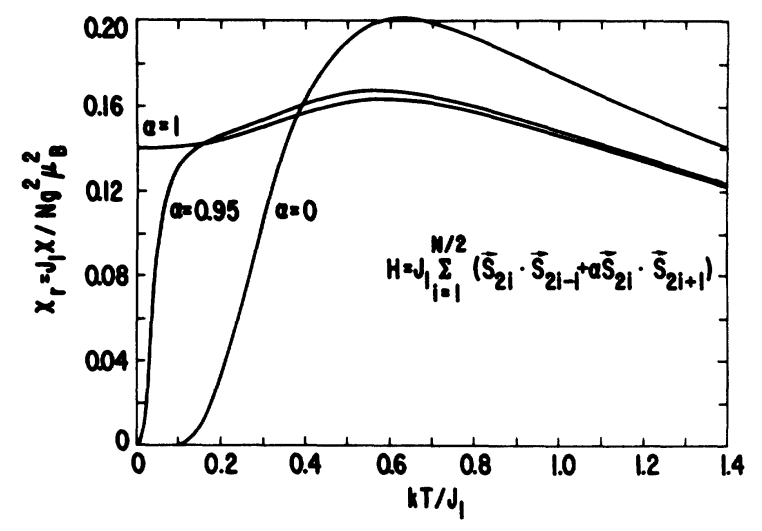

FIG. 1. Antiferromagnetic susceptibility vs temperature for the uniform Heisenberg chain $(\alpha=1)$ and a weakly alternating one $(\alpha=0.95)$, each for $N \rightarrow \infty$, calculated after Bulaevskii (Refs. 17 and 18). The curve for $\alpha=0$ applies to isolated dimerized pairs.

extreme of $\alpha$ are essentially similar at low temperatures with $\chi$ vanishing exponentially. We note also that the weakly dimerized chain $(\alpha=0.95)$ closely mimics the uniform one except at the lowest temperatures.

Another way to consider the low-temperature features of the susceptibility curves is to examine the spectrum of excited states for these cases. These are shown schematically in Fig. 2. For the uniform chain $\epsilon(k)=E-E_{0} \sim|\sin k|$. The lowest spin-wave excitation is degenerate with the ground state. (Strictly speaking, the magnetic gap vanishes as $1 / N$.) For the alternating chain, an excitation gap always exists. At absolute zero, therefore, we find zero susceptibility for $\alpha<1$. For the uniform chain, however, the $1 / N$ degeneracy implies a finite density of low-lying excited states arbitrarily close to the ground state, and correspondingly a nonzero susceptibility. Thus, a dimerization of the uniform chain will lift this degeneracy, arresting zero-point fluctuations into
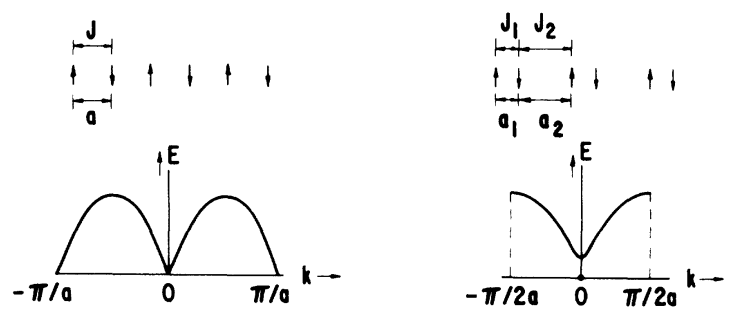

FIG. 2. Schematic representation of low-lying spinwave excitation energies vs wave vector $\vec{k}$ for a uniform Heisenberg AF chain and an alternating chain $(\alpha<1)$. For the latter, the heavy dot at $k=0$ indicates the ground state. Also, the unit cell is doubled $\left(a_{1}+a_{2}=2 a\right)$ which halves the zone-boundary wave vector. 
low-lying magnetic states, and lowering the ground-state magnetic energy. This is the essence of the spin-Peierls transition. We comment in passing on the importance of lifting the degeneracy. If we had (incorrectly) taken the frozen Néel AF ground state for the uniform chain, a linear perturbation of the exchange $\left[J_{1}=J(1+\delta) ; J_{2}\right.$ $=J(1-\delta) ; \delta \leqslant 1]$ would not have lowered the magnetic energy. [Note the relationship $\alpha=(1-\delta) /$ $(1+\delta)$.] In Fig. 3 we plot the actual ground-state free energies for dimerization of the Heisenberg, $X Y$, and Ising models of a linear AF chain. The energy lowering near the uniform limit ( $\alpha=1)$ goes like $\eta^{2} \ln \eta$ for the $X Y$ model, ${ }^{20,21}$ where $\eta$ represents lattice distortion, and like $\eta^{2} \ln ^{2} \eta$ for the Heisenberg model in a Hartree-Fock approximation. ${ }^{22,23}$ Since the lattice distortion energy increases as $\eta^{2}$, the spin-lattice system lowers its energy by distortion.

More detailed theoretical work on the spinPeierls transition was not pursued for several years after Chesnut. Pincus ${ }^{20}$ and Beni and Pincus $^{24}$ examined the $X Y$ magnetic exchange model which can be solved exactly, along with an elastic lattice and separation-dependent exchange. They treated the lattice in the adiabatic approximation neglecting nuclear motion. The calculation resulted in a second-order transition from a uniform chain to a partly dimerized chain, indicat-

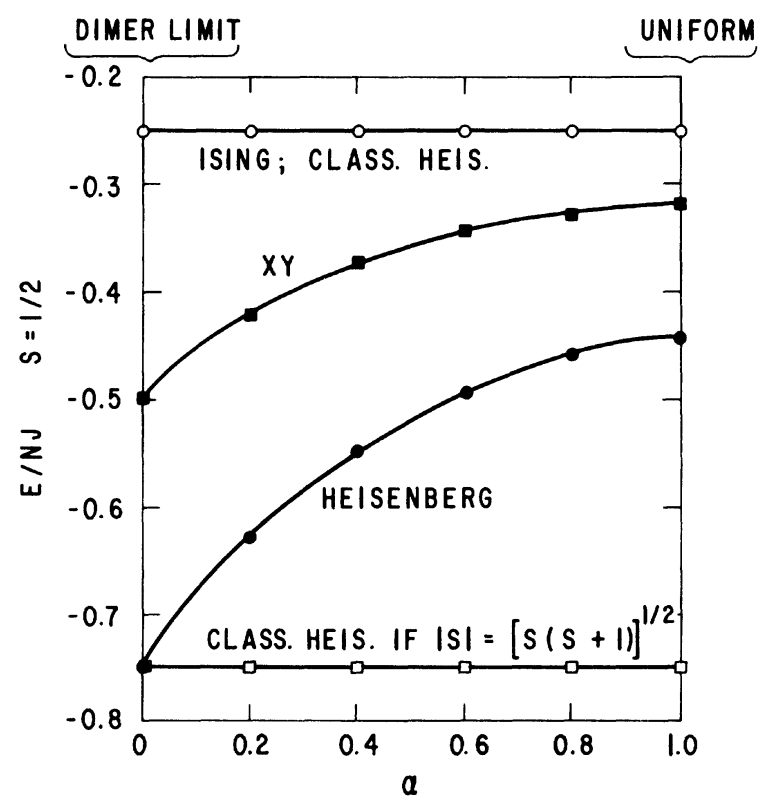

FIG. 3. Ground-state free energies of alternating magnetic chains as a function of the amount of dimerization, $\alpha=J_{2} / J_{1}$, in Eq. (1) with $J_{1}+J_{2}=2 J$, and $J_{1}, J_{2}>0$. Calculations are for the Heisenberg (after Duffy and Barr, Ref. 19), the $X Y$ (after Pincus, Ref. 20), the Ising and classical models of an AF chain. ing a temperature dependence of the alternation parameter, $\alpha$. Beni ${ }^{22}$ also considered the Heisenberg exchange-coupled chain in the Hartree-Fock approximation (after Bulaevskii ${ }^{17}$ ) and, repeating the same lattice treatment, he obtained results similar to those found for the $X Y$ model.

Recent studies by Dubois and Carton ${ }^{21}$ and by Pytte $^{23}$ have incorporated 3-D lattice dynamics in treating this problem. Dubois and Carton reexamined the $X Y$ exchange model in both a strongcoupling (between spins and lattice) and weak-coupling limit with results qualitatively the same as Pincus and Beni. They also pointed out that the instability would not occur in the Ising model. Pytte found the instability in Heisenberg chains and we recount his treatment in Sec. IV. Pytte ${ }^{25}$ also presented a more rigorous examination of an Ising 1 - D system with 3 - D lattice dynamics which explicitly involved interchain coupling. In that treatment, however, a phase transition could occur only with a special (nonlinear) form for the separation-dependent exchange.

In this historical development of the spin-Peierls model the more detailed theories have generally elaborated upon their predecessors without negating their essential features. Further, it is encouraging to note that a very recent rigorous calculation (quite distinct from the foregoing class of theories) of the time-dependent correlation functions of the continuum Heisenberg-Ising model predicts instabilities in quantum-mechanical magnetic chains. ${ }^{26}$

\section{EXPERIMENT AND RESULTS}

A. Chemical and structural

In a systematic study of donor-acceptor $(D \cdot A)$ compounds formed by the interaction of TTF with planar metal complexes, we have prepared a series of TTF - BDT derivatives where BDT represents a group of planar bis-ethylenedithiolene metal complexes of the type $\left[M \mathrm{~S}_{4} \mathrm{C}_{4} X_{4}\right]^{n}(M=\mathrm{Ni}$, $\mathrm{Pd}, \mathrm{Pt}, \mathrm{Cu}, \mathrm{Au} ; \mathrm{X}=\mathrm{H}, \mathrm{CF}_{3} ; n=0,-1$ ) (cf. Figs. 4 and 9). The BDT complexes are a well-studied class of coordination compounds known for their planar molecular geometry, delocalized $\pi$-electronic structures, reversible electron transfer behavior, and ability to form $D \cdot A$ compounds with organic donor molecules. ${ }^{27}$ In these respects, the BDT complexes are closely analogous to TCNQ, but, by virtue of their metal-organic character, are more amenable to systematic changes in electronic structure through chemical substitution. In particular, changes in both the central metal ion $M$ and the ligand substituent $X$ can be effected, producing wide variations in electron affinity and orbital occupancy, without appreciably changing 

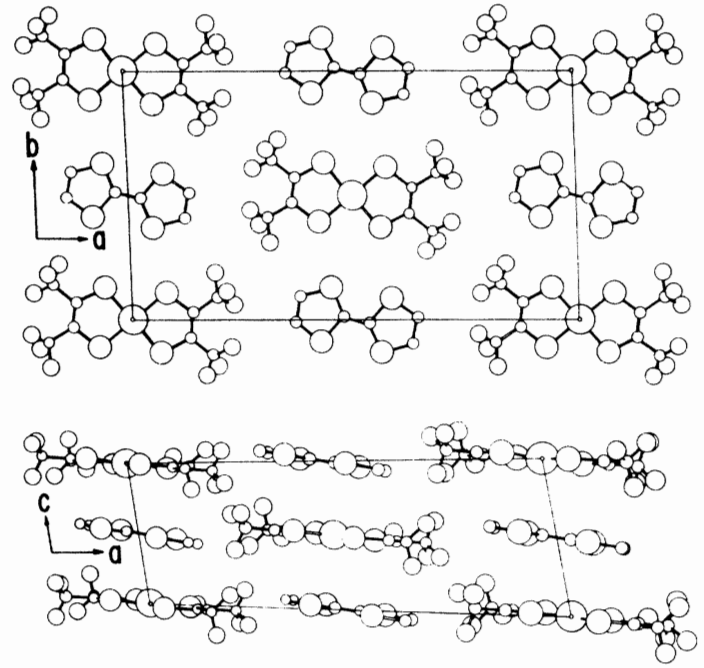

FIG. 4. View of the TTF $\bullet M \mathrm{~S}_{4} \mathrm{C}_{4}\left(\mathrm{CF}_{3}\right)_{4}, M=\mathrm{Cu}, \mathrm{Au}$, structure in the $a-b$ and $a-c$ planes. $M \mathrm{~S}_{4} \mathrm{C}_{4}\left(\mathrm{CF}_{3}\right)_{4}$ anion is at the corners and face centers. The TTF cation is at the midpoint of each edge.

the basic planar molecular geometry. We have been using this structural flexibility to investigate structure-property relationships in $D \cdot A$ compounds with TTF and have found substantial variations in solid-state packing arrangements as well as physical properties in the new $m$ TTF $\cdot n$ BDT complexes prepared. Preliminary results of our studies on the compounds, $(\mathrm{TTF})_{2} \mathrm{NiS}_{4} \mathrm{C}_{4} \mathrm{H}_{4}$, $(\mathrm{TTF})_{2}\left(\mathrm{NiS}_{4} \mathrm{C}_{4} \mathrm{H}_{4}\right)_{3}$, and TTF $\cdot M_{4} \mathrm{C}_{4}\left(\mathrm{CF}_{3}\right)_{4}(M=\mathrm{Ni}$, $\mathrm{Pt}, \mathrm{Cu}, \mathrm{Au})$ have been reported elsewhere.$^{5,6}$

The latter compounds, TTF $\cdot M \mathrm{~S}_{4} \mathrm{C}_{4}\left(\mathrm{CF}_{3}\right)_{4}$, are found to be poor electrical conductors in contrast to TTF - TCNQ but exhibit a variety of interesting magnetic behavior resulting from cooperative interactions among unpaired electrons in localized orbitals of $\pi$ symmetry on the $\mathrm{TTF}^{+}$and $\mathrm{MS}_{4} \mathrm{C}_{4}\left(\mathrm{CF}_{3}\right)_{4}^{-}$units in the structure. The magnetic properties are quite dependent on the specific arrangement of the molecular units in the structure as well as the nature of the metal ion. Singlecrystal $x$-ray diffraction studies have been used to determine the crystal structures and to relate the crystal-packing arrangement to the anisotrop- ic magnetic properties observed. These complexes all crystallize in triclinic cells with an alternate stacking arrangement of $\mathrm{TTF}^{+}$and $M_{4} \mathrm{C}_{4}\left(\mathrm{CF}_{3}\right)_{4}^{-}$units along the $c$ axis of the structure (Table I). The $M=\mathrm{Pt}, \mathrm{Cu}$, and $\mathrm{Au}$ compounds are is ostructural and assume the NaCl-like packing arrangement shown in Fig. 4.

The molecular planes of both kinds of ions are nearly parallel to the (001) planes, and the protrusion of the $\pi$ orbitals from these molecular planes presumably favors electronic interactions along the $c$ axis. The alternate stacking arrangement and large separation ( $3.9 \AA)$ implies highly localized electronic orbitals, a fact borne out by the conductivity, which is less than $10^{-9}(\Omega \mathrm{cm})^{-1}$ by four-probe dc measurements on single crystals. We identify this $c$-axis stack as a chain unit, although other evidence is desirable.

In the case of the $\mathrm{Pt}$ derivative, both ions carry a spin and the dominant intrachain magnetic interactions at high temperatures are apparently of the direct-exchange type and ferromagnetic in character. ${ }^{6}$ On the other hand, for $M=\mathrm{Cu}, \mathrm{Au}$, the additional electron in the corresponding $M \mathrm{~S}_{4} \mathrm{C}_{4}\left(\mathrm{CF}_{3}\right)_{4}{ }^{-}$ units fills the highest occupied molecular orbital to give a diamagnetic anion, leaving unpaired spins only on the $\mathrm{TTF}^{+}$units in the structure. The compounds with $M=\mathrm{Cu}, \mathrm{Au}$ are the focal point of this paper.

The structural views shown in Fig. 4 were obtained in detail for the $M=\mathrm{Pt}$ compound at room temperature. Detailed studies on the others over a broad range of temperatures are underway. While the latter studies may throw light on differences between the several compounds, there is enough similarity (Table I) to permit us to use the structure shown.

The physical property measurements signaled an anomaly at $250 \mathrm{~K}(200 \mathrm{~K})$ for the $\mathrm{Cu}(\mathrm{Au})$ compound. Preliminary $\mathrm{x}-\mathrm{ray}$ studies in this temperature region indicate the basic crystal structure is unaltered although abrupt changes in several of the lattice parameters were observed at these temperatures.

The TTF $\cdot M \mathrm{~S}_{4} \mathrm{C}_{4}\left(\mathrm{CF}_{3}\right)_{4}$ complexes were obtained by metathesis of the $\mathrm{TTF}^{+} \mathrm{Cl}^{-}$and the tetra-alkyl

TABLE I. Unit-cell ${ }^{a}$ data for isostructural 1:1 TTF $\cdot M \mathrm{~S}_{4} \mathrm{C}_{4}\left(\mathrm{CF}_{3}\right)_{4}$ charge-transfer complexes.

\begin{tabular}{ccccccc}
\hline \hline Compound & \multicolumn{1}{c}{$a$} & $b$ & $c$ & $\alpha$ & $\beta$ & $\gamma$ \\
\hline $\mathrm{TTF} \cdot \mathrm{CuS}_{4} \mathrm{C}_{4}\left(\mathrm{CF}_{3}\right)_{4}$ & $23.1 \AA$ & $13.2 \AA$ & $7.80 \AA$ & $92.7^{\circ}$ & $101.8^{\circ}$ & $90^{\circ}$ \\
$\mathrm{TTF} \cdot \mathrm{AuS}_{4} \mathrm{C}_{4}\left(\mathrm{CF}_{3}\right)_{4}$ & 23.27 & 13.04 & 7.86 & $90.9^{\circ}$ & $102.5^{\circ}$ & $91.2^{\circ}$ \\
$\mathrm{TTF} \cdot \mathrm{PtS}_{4} \mathrm{C}_{4}\left(\mathrm{CF}_{3}\right)_{4}$ & 23.34 & 13.10 & 7.82 & $90.6^{\circ}$ & $101.4^{\circ}$ & $92.0^{\circ}$ \\
\hline \hline
\end{tabular}

\footnotetext{
${ }^{\text {a }}$ The true space group is $P \overline{1}$ with one formula unit per cell but for convenience we use a
} face-centered cell with $Z=4$ and space group $F \overline{1}$. 
ammonium or tetraphenyl arsonium salts of $\mathrm{MS}_{4} \mathrm{C}_{4}\left(\mathrm{CF}_{3}\right)_{4}{ }^{-}$in methanol solution. Recystallization of the products of these reactions by slow cooling of acetonitrile solutions gave needlelike crystals, 2-3 mm long and $0.05 \times 0.3 \mathrm{~mm}^{2}$ in cross section.

\section{B. Magnetic susceptibility}

The static susceptibility was measured on both the $\mathrm{Cu}$ and $\mathrm{Au}$ complexes between 2.5 and $300 \mathrm{~K}$ with the Faraday method and supplemented, for the Au complex, by measurement between 1.6 and $4 \mathrm{~K}$ using a SQUID (superconducting quantum-interference device) magnetometer system.

The Faraday method used an electrobalance (Cahn, Model RG) and a split superconducting solenoid. The solenoid was wound from $\mathrm{Nb}-\mathrm{Ti}$ multifilamentary wire to minimize hysteresis in the field-current relationship. The split solenoid allowed the field and field gradient to be varied independently; the data were taken by setting a series of fields (10-40 kOe) and determining the force as a function of gradient at each field.

The sample of the copper complex consisted of several tens of single crystals (total mass 2.18 $\mathrm{mg}$ ) which were aligned with their long axes ( $c$ axes) parallel in a tubular holder (mass $\sim 5 \mathrm{mg}$ ) constructed from $0.005-\mathrm{cm}$-thick polycarbonate sheet and polystyrene glue. The geometry of the holder allowed a rough alignment of the $c$ axis with respect to the field. The magnetization $M$ was found to be a linear function of field (10-40 kOe) at all temperatures outside the range 6-12 $\mathrm{K}$. In the latter range, $M(H)$ was slightly concave upward and the low-field susceptibility was inferred by extrapolation. Within the accuracy of the method the susceptibility was also found to be approximately isotropic for measurements parallel and perpendicular to the $c$ axis of the bundle. The zero of the susceptibility scale was obtained from the integrated $\mathrm{EPR}$ intensity at $4 \mathrm{~K}$ relative to its value at $50 \mathrm{~K}$, as we describe below.

Data for the two directions of measurements are shown in Fig. 5. The solid lines are calculated from theory described in Sec. IV. The shift to a dashed line above $250 \mathrm{~K}$ reflects the sensitivity of the magnetic system to the transition noted in Sec. III A.

The Faraday-method sample of the gold complex $(2.92 \mathrm{mg}$ ) was mounted similarly to the copper but the plastic holder tube was machined from solid polycarbonate. In contrast to the tube made from polycarbonate film, this one yielded a temperature-dependent background. The measured susceptibility of the holder and a calculated diamagnetic contribution of the complex were subtracted

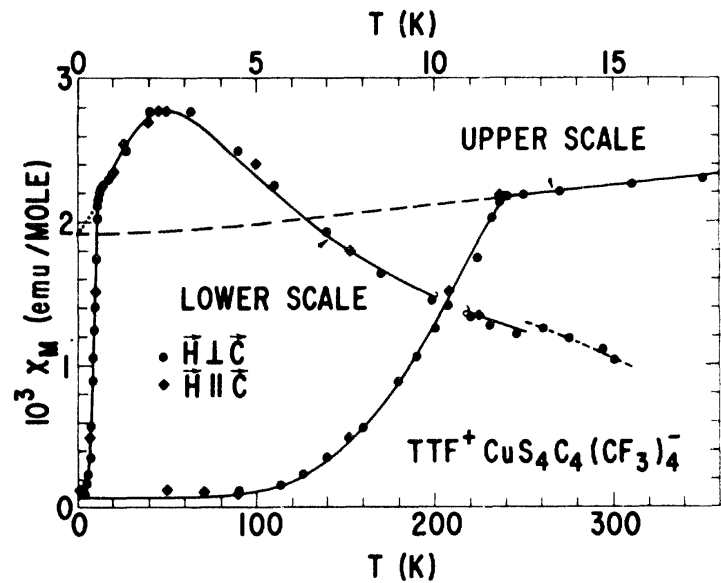

FIG. 5. Magnetic susceptibility of TTF $\cdot \mathrm{CuS}_{4} \mathrm{C}_{4}\left(\mathrm{CF}_{3}\right)_{4}$ along two directions. Solid lines are calculated from a spin-Peierls theory which contains AF chains with uniform exchange above $12 \mathrm{~K}$ and temperature-dependent alternating exchange below.

in order to obtain the spin susceptibility presented in Fig. 6. The solid lines are obtained from a fit to theory described in Sec. IV. The crystals of the gold complex cracked into small pieces upon temperature cycling. This behavior may be associated with the crystal transition observable at $200 \mathrm{~K}$.

The SQUID magnetometer was a modification of that described by Gollub et al., ${ }^{28}$ utilizing an $\mathbf{r f}$ SQUID in place of the dc double-point-contact SQUID of the original. The sample crystals (mass of $19.2 \mathrm{mg}$ ) and a small amount of Apiezon $\mathrm{N}$ grease for thermal contact were mounted in a small high-purity copper can in an evacuated

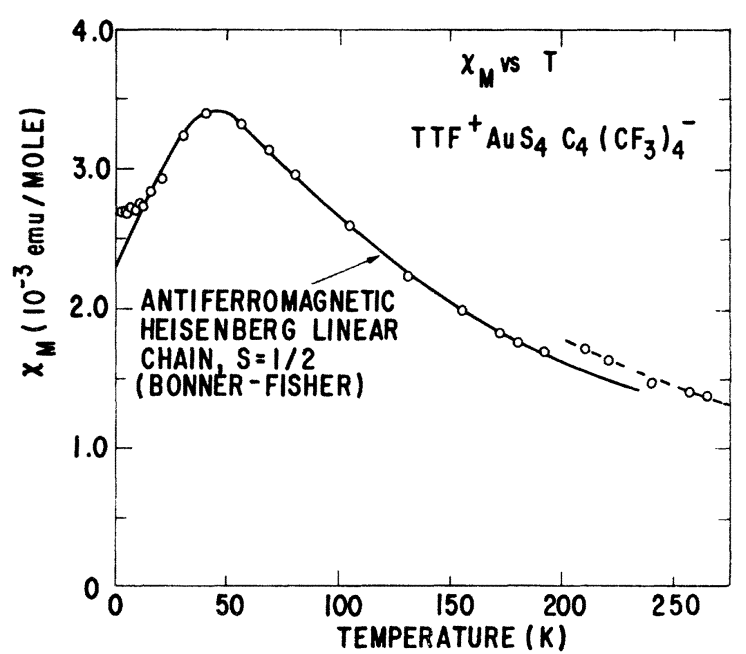

FIG. 6. Magnetic susceptibility of TTF $\cdot \mathrm{AuS}_{4} \mathrm{C}_{4}\left(\mathrm{CF}_{3}\right)_{4}$ powder for $T>2.5 \mathrm{~K}$. The solid line is the BonnerFisher (Ref. 16) calculation for a uniform AF chain, $S$ $=\frac{1}{2}$. 
chamber. The temperature of the can was varied, and changes of the sample's magnetic moment were recorded continuously as a function of temperature, as measured with a germanium resistance thermometer. A separate background run was also made with the copper can empty to ascertain the background signal, which was subtracted to obtain the sample signal. The background signal was less than $10 \%$ of the sample signal. The magnetic field used was $10 \mathrm{Oe}$, with a check at 100 Oe to confirm the field independence of the susceptibility.

The magnetometer was calibrated by measuring a crystal of the paramagnetic salt chrome-potassium alum. The zero of the sample susceptibility (only changes were measured) was obtained by matching the SQUID results at $2.75 \mathrm{~K}$ to the integrated EPR intensity described in Sec. III C. The scale of the integrated EPR intensity was determined from the Faraday method data. All these data are presented in Fig. 7, combining those of Fig. 6 with the SQUID and EPR results.

\section{Electron paramagnetic resonance (EPR)}

EPR measurements were performed on a balanced bolometer spectrometer at $20 \mathrm{GHz}$. (The sample was mounted on a quartz rod in the center of a $\mathrm{TE}_{011}$ cylindrical cavity.) Resonance was observed in absorption with the power level adjusted as necessary to prevent saturation of the resonance. The temperature was varied from 1.45 to $4.2 \mathrm{~K}$ and 10 to $20.4 \mathrm{~K}$ by pumping on liquid helium or hydrogen, respectively, surrounding the cavity. Above $20.4 \mathrm{~K}$, the coolant was expelled and the temperature measured by a copper-Constanstan thermocouple mounted on the cavity.

For intensity measurements, $94-\mathrm{Hz}$ square-

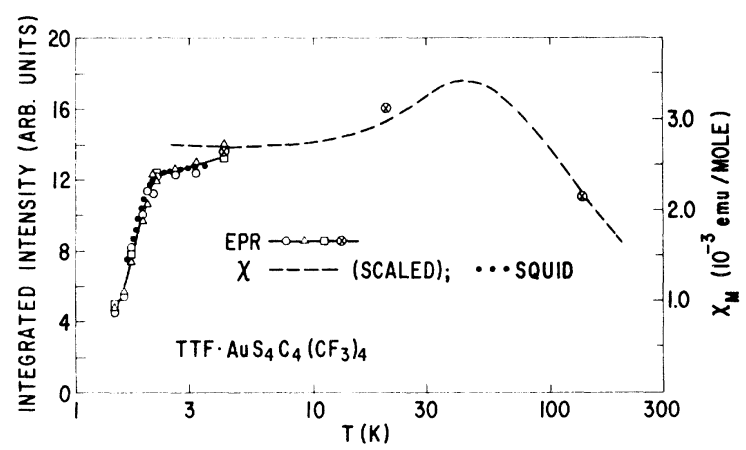

FIG. 7. Low-temperature powder magnetic susceptibility (SQUID magnetomet $r$ ) and integrated EPR intensity with $H$ along two major crystal axes for

TTF $\cdot \mathrm{AuS}_{4} \mathrm{C}_{4}\left(\mathrm{CF}_{3}\right)_{4}$. These are scaled to match the static Faraday susceptibility results of Fig. 6 at $4 \mathrm{~K}$. The resonance spectrometer sensitivity is calibrated for all data shown. wave source modulation was used, the recorded signal after lock-in detection plotting out the absorption signal directly. ${ }^{29}$ The intensity of the signal was then estimated by integrating the area under the recorded curves. Absolute intensity measurements were not attempted. Also, in most of the studies no correction was made for possible changes in the spectrometer sensitivity versus temperature. These can occur due to changes in losses in the sample and due to conductivity changes in the brass cavity (i.e., a change in $Q$ of the cavity). Relatively small changes in the coupling of the cavity to the waveguide were observed over the temperature range studied, and these effects, therefore, should not be large. (In one case to be described, spectrometer sensitivity was checked versus temperature using the method of Silsbee, ${ }^{30}$ where a small frequency modulation is imposed upon the klystron and the resulting calibration signal observed in dispersion. The spectrometer sensitivity was found to decrease somewhat with increasing temperature. At $140 \mathrm{~K}$, the sensitivity was $\sim 60 \%$ that at $4.2 \mathrm{~K}$.)

For a single crystal of the copper complex, a single strong E PR transition was observed for $T$ $\geqslant 10 \mathrm{~K}$. (Other weaker lines were observed at $T$ $<4.2 \mathrm{~K}$.) For the gold complex, a single strong EPR transition was observed over the full region $T \geqslant 1.45 \mathrm{~K}$. For each, the angular dependence is described by the spin Hamiltonian

$$
H=\mu_{B} \overrightarrow{\mathrm{S}} \cdot \overrightarrow{\mathrm{g}} \cdot \overrightarrow{\mathrm{H}}
$$

where $S=\frac{1}{2}, \overrightarrow{\mathrm{g}}$ is the magnetogyric tensor, and $\mu_{B}$ is the Bohr magneton. This dominant transition in each case arises from the $\mathrm{TTF}^{+}$molecular unit, as identified below.

For accurate determination of the principal axes of the $g$ tensor, two crystals were mounted to form a pseudotwin, side by side, with their $a^{*}$ axes parallel, but with the $c$ axis of one aligned $180^{\circ}$ from the $c$ axis of the other. (These axes are easily identified from the crystal morphology.) Studies with $\overrightarrow{\mathrm{H}}$ in the $a^{*} c$ and $a^{*} b$ planes therefore produce two lines which in their angular dependence are identical except that they are shifted by $2 \theta$, where $\theta$ is the angle between the extreme values of $g$ in that plane and the $a^{*}$ axis.

Our EPR study of the TTF $\cdot$ BDT complexes gives several kinds of information. It complements and extends the static magnetic results because the integrated absorption intensity is proportional to the susceptibility. It probes the electronic structure of the spin carriers through the principal $g$ values and relates them structurally to the lattice through the orientation of the axes of the $\ddot{g}$ tensor. Lastly, it contributes to the study of spin dynam- 


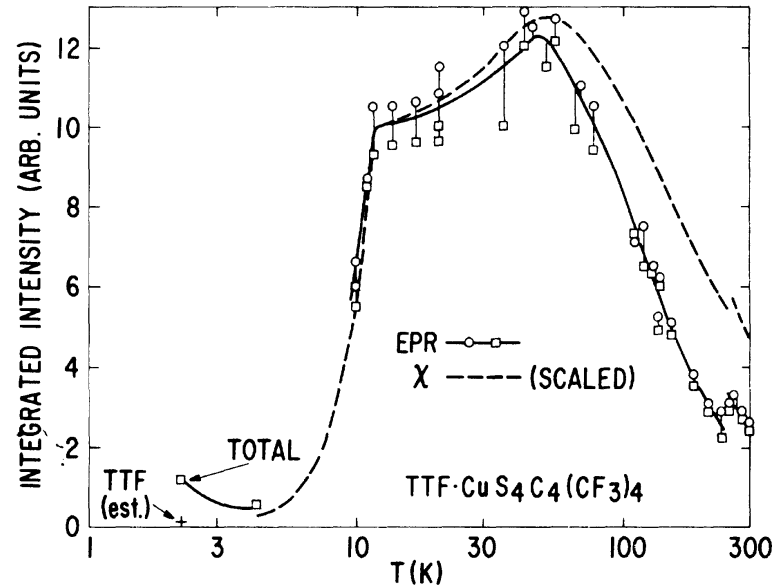

FIG. 8. Integrated EPR intensity vs temperature for $\mathrm{TTF} \cdot \mathrm{CuS}_{4} \mathrm{C}_{4}\left(\mathrm{CF}_{3}\right)_{4}$ for two perpendicular crystal orientations $(\overrightarrow{\mathrm{H}}\|\overrightarrow{\mathrm{c}}, \bigcirc ; \overrightarrow{\mathrm{H}}\| \overrightarrow{\mathrm{b}}, \square)$. The dashed line is the susceptibility taken from Fig. 5, scaled to fit at $12 \mathrm{~K}$. For $T \geq 10 \mathrm{~K}$ the intensity is from a single line associated with the $\mathrm{TTF}^{+}$ion. For $T \leq 4 \mathrm{~K}$, several species contribute. "+" sign estimates the contribution of residual $\mathrm{TTF}^{+}$at $2.3 \mathrm{~K}$.

ics in coupled spin systems (of low dimensionality) through the behavior of the absorption linewidth.

In Fig. 8 we show the integrated intensity versus temperature for $\mathrm{TTF} \cdot \mathrm{CuS}_{4} \mathrm{C}_{4}\left(\mathrm{CF}_{3}\right)_{4}$ for two differerent crystal orientations. The intensity is isotropic within the experimental accuracy. Also shown for comparison is a curve repeating the static susceptibility data of Fig. 5 . As noted above, we do not attach significance to the difference between the two sets of data at higher temperatures but consider them to be qualitatively the same. At these temperatures the static data are more accurate. At the lowest temperature $(2.3 \mathrm{~K})$ a variety of paramagnetic species contributes to the residual absorption. The new ones differ in their principal $g$ values from the domin- (c)

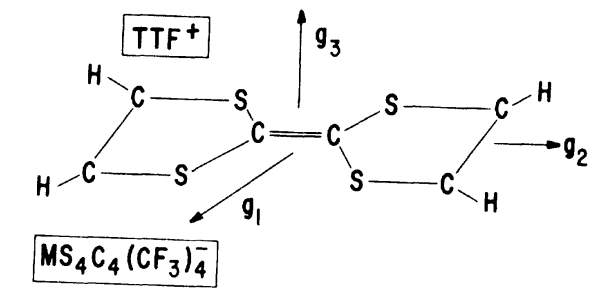

(b)<smiles>C[As](SC(C(F)(F)F)C(F)(F)F)C(C(F)(F)F)C(F)(F)F</smiles>

(a)

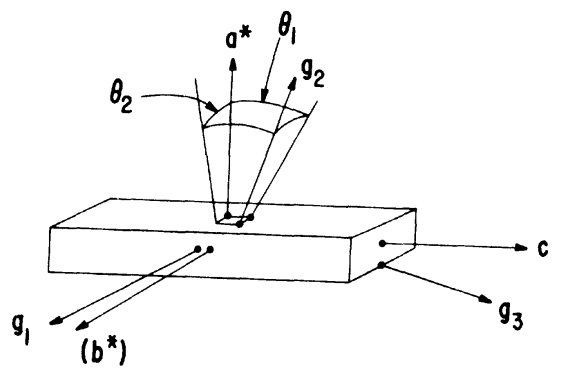

FIG. 9. Schematic representation of the $\mathrm{TTF}^{+}$and $M \mathrm{BDT}^{-}$ions showing principal axes of their respective $g$ tensors. Part (a) defines the angles between the crystal axes and the $g$ tensor axes. It was found experimentally that $g_{3}$ lies in the $a^{*}-c$ plane.

ant component at $T \geqslant 10 \mathrm{~K}$. As noted above (Sec. III B), we use the low-temperature intensity behavior to fix the zero of the static susceptibility.

In Table II we present the principal $g$-tensor values measured on the $\mathrm{Cu}$ compound, referred to orientations of the crystal as shown in Fig. 9(a).

The first $(A)$ is the dominant spectrum at $T$ $\geqslant 10 \mathrm{~K}$. Also given are $g$ values previously reported for $\mathrm{TTF}^{+}$. The close similarity confirms the identification of this spectrum as arising from

TABLE II. Principal $g$ values and axes observed in $\mathrm{TTF} \cdot \mathrm{CuS}_{4} \mathrm{C}_{4}\left(\mathrm{CF}_{3}\right)_{4}$. Axes and angles are shown in Fig. 9(a).

\begin{tabular}{crcccccc}
\hline \hline Spectrum & $T(\mathrm{~K})$ & Ion & $g_{1}$ & $g_{2}$ & $g_{3}$ & $\theta_{1}$ & $\theta_{2}$ \\
\hline \multirow{2}{*}{$A$} & 20.4 & $\mathrm{TTF}^{+\mathrm{a}}$ & 2.0069 & 2.0151 & 2.0016 & $21.6^{\circ}$ & $4.5^{\circ}$ \\
$B$ & 2.3 & $?$ & 2.0062 & 2.0178 & 2.0085 & $26.5^{\circ}$ & $\left(0 \pm 10^{\circ}\right)$ \\
$C$ & 2.3 & $M \mathrm{~S}_{4} \mathrm{C}_{4}\left(\mathrm{CF}_{3}\right)_{4}{ }^{\mathrm{b}}$ & 2.1236 & 2.0344 & 1.9903 & $15.7^{\circ}$ & $3.2^{\circ}$ \\
\hline \hline
\end{tabular}

${ }^{a}$ The principal $g$ values for $\mathrm{TTF}^{+}$in TTF $\cdot$ TCNQ have been reported by W. M. Walsh, Jr., L. W. Rupp, Jr., F. Wudl, D. E. Shaefer, and G. A. Thomas [Bull. Am. Phys. Soc. 19, 296, (1974) ] to be $2.0078,2.0116,2.0021$. Y. Tomkiewicz, F. Mehran, D. C. Green and B. A. Scott [ibid. 19, $334(1974)]$ report an axially symmetric spectrum in TTF $\cdot \mathrm{Cl}$ with $g_{\perp}=2.011$, $g_{11}=2.002$.

${ }^{b}$ Presumably either $\mathrm{NiS}_{4} \mathrm{C}_{4}\left(\mathrm{CF}_{3}\right)_{4}{ }^{-}$or $\mathrm{CuS}_{4} \mathrm{C}_{4}\left(\mathrm{CF}_{3}\right)_{4}{ }^{0}$; see text. Principal $g$ values for $\mathrm{NiS}_{4} \mathrm{C}_{4}$ $\left(\mathrm{CF}_{3}\right)_{4}{ }^{-}$have been measured by A. Davison, N. Edelstein, R. H. Holm, and A. H. Maki [Inorg. Chem. $\underline{3}, 814(1964)]$ to be $2.137,2.044,1.996$. 
the $\mathrm{TTF}^{+}$molecular unit, and therefore the role of this ion as the dominant spin carrier in our system. The second two spectra $(B, C)$ are two of the dominant but weak paramagnetic spectra (i.e., intensities increase with decreasing temperature) that emerge at the lowest temperature. The $g$ values for spectrum $C$ are observed to be similar to those reported for the $\mathrm{NiS}_{4} \mathrm{C}_{4}\left(\mathrm{CF}_{3}\right)_{4}{ }^{-}$ion (also given in the table) suggesting that it may arise from an impurity or possibly from the isoelectronic $\mathrm{CuS}_{4} \mathrm{C}_{4}\left(\mathrm{CF}_{3}\right)_{4}{ }^{0}$ which could be present as a structural or stoichiometric defect. Spectrum $B$ is not identified. At $2.3 \mathrm{~K}$ there also remains a small amount of the $\mathrm{TTF}^{+}$spectrum (see Fig. 8) resulting from a "defect" (e.g., chain end) that did not take part in the freeze-out of active spins.

The angles listed in Table II define the orientation of the paramagnetic molecular complexes with respect to the crystal axes. For spectra $A$ and $C$, they are remarkably close to the tilt angles for the corresponding $\mathrm{TTF}^{+}$and $\mathrm{MS}_{4} \mathrm{C}_{4}\left(\mathrm{CF}_{3}\right)_{4}{ }^{-}$ions shown in Fig. 4, which represents the room-temperature structure for the Pt compound. In Fig. 9, we therefore also show the $g$-tensor axes with respect to the implied molecular framework. A more detailed comparison awaits the results of structural studies on the $\mathrm{Cu}$ (and $\mathrm{Au}$ ) compound. The temperature dependence of the tilt of the $\mathrm{TTF}^{+}$ion in the (010) plane as measured by EPR on the Cu compound is shown in Fig. 10(a). The significant features are the sharp change at the $250-\mathrm{K}$ transition and the absence of any observable change of note in the region of 10 to $12 \mathrm{~K}$, despite a careful search. (At $4 \mathrm{~K}$, competing contributions prevent a meaningful analysis.) In Fig. 10(b), the tilt temperature dependence is shown for the (001) plane for the temperature range 130 to $270 \mathrm{~K}$.

The temperature dependence of the peak-to-peak derivative linewidths with $\overrightarrow{\mathrm{H}}$ along the $b\left(\sim g_{1}\right)$ and $c\left(\sim g_{3}\right)$ axes is shown in Fig. 11 for the $\mathrm{Cu}$ compound. For $T \geqslant 10 \mathrm{~K}$, the spectrum arises solely from $\mathrm{TTF}^{+}$. Again, the $250-\mathrm{K}$ transition is manifest. The broken line is a normalized plot of $\chi T$, to be discussed in Sec. IV. At $4.2 \mathrm{~K}$, a single broad resonance is observed that arises from both the residual $\mathrm{TTF}^{+}$spectrum and that labeled $B$ in Table II, which overlap. Upon lowering the temperature, the lines sharpen and the individual spectra emerge, along with spectrum $C$, as shown.

Similar measurements were carried out for $\mathrm{TTF} \cdot \mathrm{AuS}_{4} \mathrm{C}_{4}\left(\mathrm{CF}_{3}\right)_{4}$ but in less detail. Throughout the complete temperature range, a single strong anisotropic EPR line was observed. A somewhat uncertain crystal morphology prevented a precise determination of the principal axes of the $g$ tensor although values close to those of $\mathrm{TTF}^{+}$in Table II
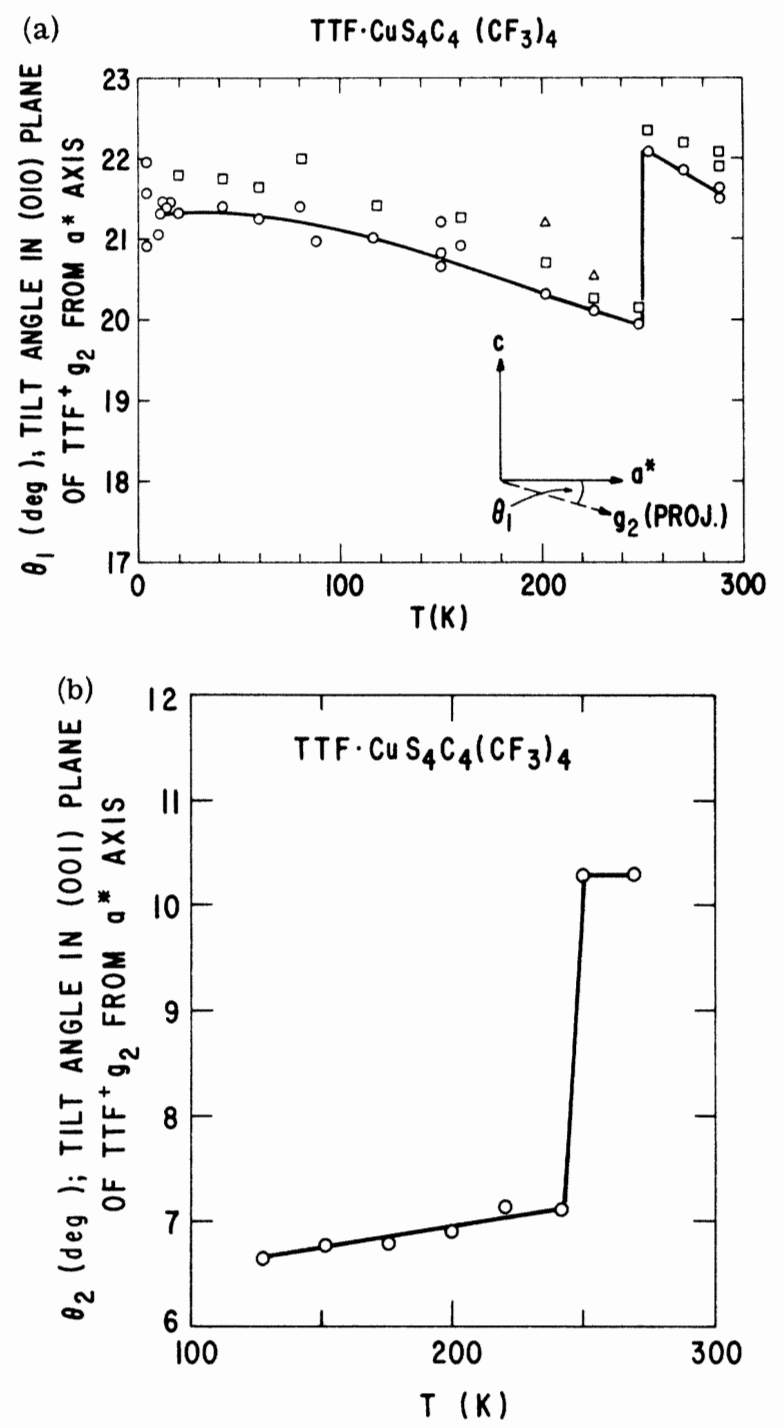

FIG. 10. (a) Temperature dependence of the tilt of the $\mathrm{TTF}^{+}$ion in the (010) plane of TTF $\cdot \mathrm{CuS}_{4} \mathrm{C}_{4}\left(\mathrm{CF}_{3}\right)_{4}$, measured by $\theta_{1}$ in EPR, cf. Fig. 9; (b) similar tilt in the (001) plane measured by $\theta_{2}$.

were obtained. Also a clear shift in axes at the 200-K transition was seen despite the uncertain knowledge of the tilt angle. In Fig. 7 is shown the integrated intensity versus temperature. Only those data are shown for which the spectrometer sensitivity was calibrated. The agreement with the static susceptibility (dashed curve) is satisfactory. (More data in the uncalibrated state confirm the peak and general overall shape.) These results and the SQUID data from 4 down to $1.5 \mathrm{~K}$ clearly show behavior similar to that found at and below $12 \mathrm{~K}$ in the $\mathrm{Cu}$ compound. In both cases the susceptibility is "freezing-out" rapidly, but continuously. In Fig. 12 the linewidth (peak-to-peak derivative) is shown as a function of temperature 


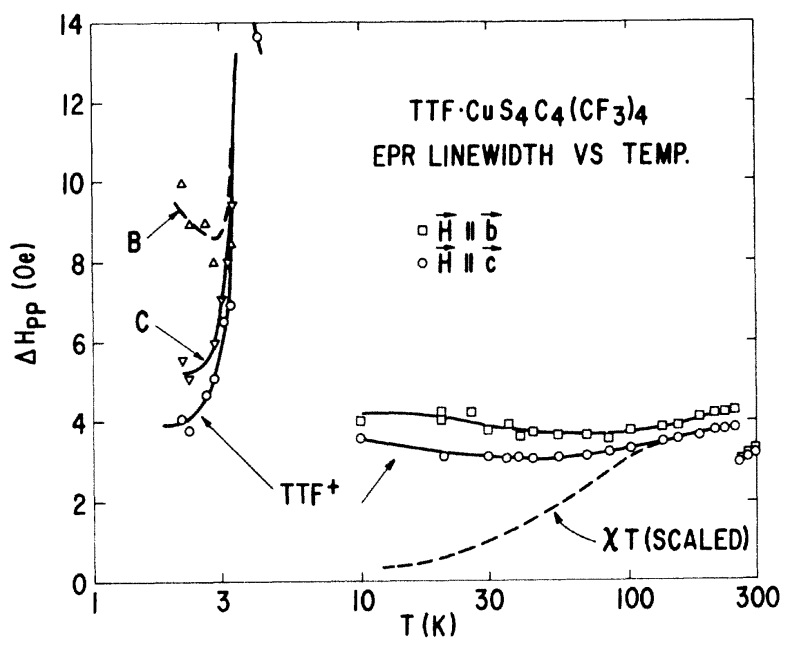

FIG. 11. EPR peak-to-peak derivative linewidth, $\Delta H$, vs temperature at $20 \mathrm{GHz}$ for TTF $\cdot \mathrm{CuS}_{4} \mathrm{C}_{4}\left(\mathrm{CF}_{3}\right)_{4}$ for $\overrightarrow{\mathrm{H}}$ parallel to two major crystal axes. The broken line is a comparative plot of $\chi T$ normalized to $\Delta H$ at $250 \mathrm{~K}$. Below $4.2 \mathrm{~K}$, the results shown are for $\overrightarrow{\mathrm{H}} \| \overrightarrow{\mathrm{c}}$ only.

from 4 to $250 \mathrm{~K}$, again in comparison with a normalized curve of $\chi T$. These data also show a clear break at the $200-\mathrm{K}$ transition.

\section{DISCUSSION AND ANALYSIS}

\section{A. Introductory overview}

Considering the results of Sec. III, one may first ask if the compounds studied are well described as Heisenberg antifer romagnetic linear chains. The Heisenberg character of the exchange can be inferred by the very small shifts observed in the EPR $g$ values versus temperature. If anisotropic components exist in the spin-spin coupling (ex-

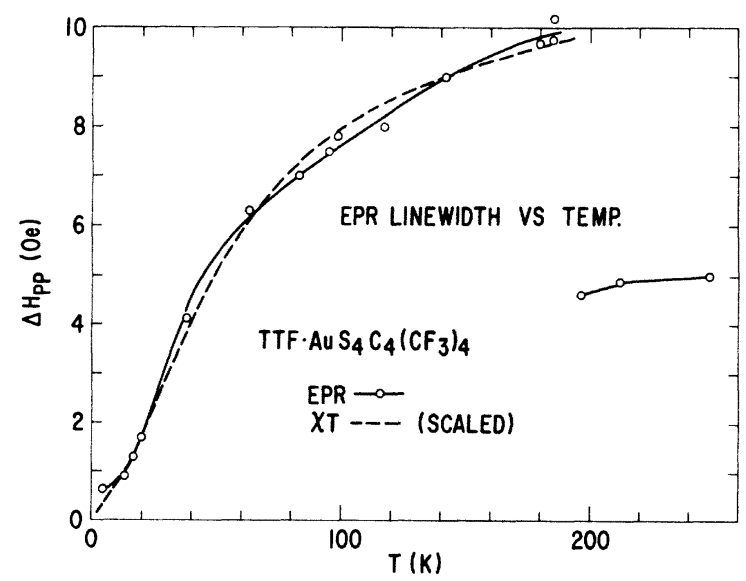

FIG. 12, EPR peak-to-peak derivative linewidth $\Delta H$ at $20 \mathrm{GHz}$ vs temperature for TTF $\cdot \mathrm{AuS}_{4} \mathrm{C}_{4}\left(\mathrm{CF}_{3}\right)_{4}$. Dashed line is a comparative plot of $\chi T$ normalized at $180 \mathrm{~K}$. change and/or dipole-dipole), shifts in the $\mathrm{TTF}^{+}$ $g$ values will occur proportional to $\left\langle S_{l}^{z} S_{l+1}^{z}\right\rangle /\left\langle S^{z}\right\rangle$, reflecting the setting in of long- and short-range order. ${ }^{31}$ The observed shifts are small $(\Delta g$ $<0.001$, occurring mainly in the $10-20 \mathrm{~K}$ region for the copper compound), indicating that the principal exchange mechanism must be Heisenberg (isotropic) in character. While the maximum in the susceptibility suggests AF interactions, more convincing evidence is found in the behavior of $\chi T$ which decreases with decreasing temperature. (A Curie-Weiss plot also shows a negative intercept, but is less reliable because the asymptotic high-temperature region is not attained.)

The question of magnetic dimensionality, however, requires more careful reasoning. From structural considerations, the usual suggestive feature ${ }^{4}$ are distances between magnetic ions (in different directions), directionality of exchange or super-exchange paths between spins, and special alignment of orbitals. The alignment of $\pi$ orbitals of the $\mathrm{TTF}^{+}$and $\mathrm{BDT}^{-}$ions, protruding perpendicular to the planar molecule ions which are themselves nearly parallel to (001) planes (Fig. 4), strongly favors electronic interactions along the $c$ axis and conditions our choice of the "mixed" stack as a linear chain unit. Support for this may be found in the apparent absence of interchain magnetic ordering as discussed later. The broad susceptibility maximum is indicative of an AF linear chain, AF dimers, or a two-dimensional AF system. The relatively gradual drop in susceptibility below the maxima in Figs. 5 and 6 rules out dimers. The overall shapes of the curves of $\chi$ vs $T$ are very well-known for 1 - D systems, ${ }^{16}$ but only approximately for 2-D Heisenberg systems. ${ }^{4}$ Near and above their maxima, they are not very readily distinguished. Putting aside the "ideal" low-D models with no ordering temperatures, we examine the "real" Heisenberg AF systems $\left(S=\frac{1}{2}\right)$ with interchain coupling that do show ordering. An empirical monitor would be the ratio of the magnetic ordering temperature to that of the susceptibility maximum. This ratio is 0.9 or higher for $3-\mathrm{D}$ systems, about 0.5 down to 0.25 for $2-\mathrm{D}$ systems, and about 0.1 or less for good 1-D systems (some cases a bit higher). On this basis, neither the $\mathrm{Au}$ nor the $\mathrm{Cu}$ compounds show magnetic ordering in the $2-D$ and $3-D$ regimes.

Next we note that the extrapolated zero-temperature susceptibility from temperatures above the second-order transition has, for the $\mathrm{Cu}$ compound, almost exactly the ratio of $\chi(0) / \chi_{\max } \approx 0.68$ predicted by an equivalent extrapolation of the BonnerFisher ${ }^{16}$ calculation for $1-D$ systems. The latter is much preferable to the Hartree-Fock calculation of Bulaevskii used for pedagogical reasons in 
Fig. 1. (An equivalent prediction of the ratio does not exist for 2-D systems.)

Other tools for investigating dimensionality include the magnetic specific-heat behavior. The absence of a truly diamagnetic analog, plus the large lattice contribution of the multiatom molecules makes this of doubtful utility, even if it were available. The ultimate tool for this question is that of neutron scattering. Pending the outcome of such experiments, we believe from the evidence above, that the TTF $\cdot M \mathrm{~S}_{4} \mathrm{C}_{4}\left(\mathrm{CF}_{3}\right)_{4}(M=\mathrm{Cu}, \mathrm{Au})$ compounds have predominantly $1-\mathrm{D}$ magnetic interactions.

In Fig. 5 for the $\mathrm{Cu}$ compound, the solid line between 12 and $250 \mathrm{~K}$ is obtained from the BonnerFisher calculations with $J / k_{B}=77 \mathrm{~K}$ and $g=1.97$. The difference between this $g$ and those found by EPR is within the error band of the susceptibility measurement. The overall fit, obtained by a graphical method, is very good for temperatureindependent parameters. We show a similar fit for the Au compound in Fig. 6. The agreement is excellent down to $12 \mathrm{~K}$ with $g=2.05$ and $J / k_{B}$ $=68 \mathrm{~K}$. Below $12 \mathrm{~K}$, there is an apparent excess $\chi$ which may arise from impurities or brokenchain effects. ${ }^{18}$

The small deviations between the solid line and the data in Fig. 5 may be connected with a temperature dependence of $J$ as a manifestation of spin-lattice coupling. From graphical comparisons of $\chi T$ vs $T$ or $k T / J$ (experiment or theory, respectively), we have estimated $J(T)$ for the $\mathrm{Cu}$ compound in the interval $12<T<250 \mathrm{~K}$, assuming $g=2.00$ for all temperatures. The results shown in Fig. 13 give a qualitative indication for $J(T)$. The small slow decrease of $J$ between 12 and 100 $\mathrm{K}$ would be expected from thermal expansion. Above $150 \mathrm{~K}$ the method has diminished accuracy restricting further interpretation.

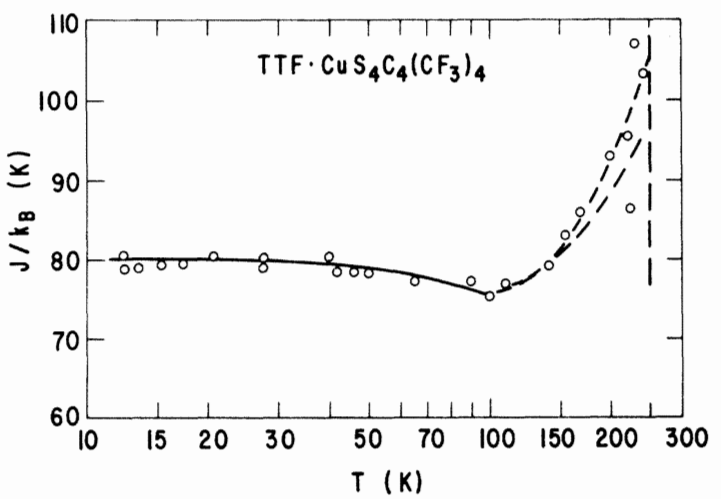

FIG. 13. Estimated temperature dependence of the AF exchange, $J(T) / k_{B}$, for TTF $\cdot \mathrm{CuS}_{4} \mathrm{C}_{4}\left(\mathrm{CF}_{3}\right)_{4}$ in the uniform chain region $(12<T<250 \mathrm{~K})$, assuming $g=2.00$ throughout.

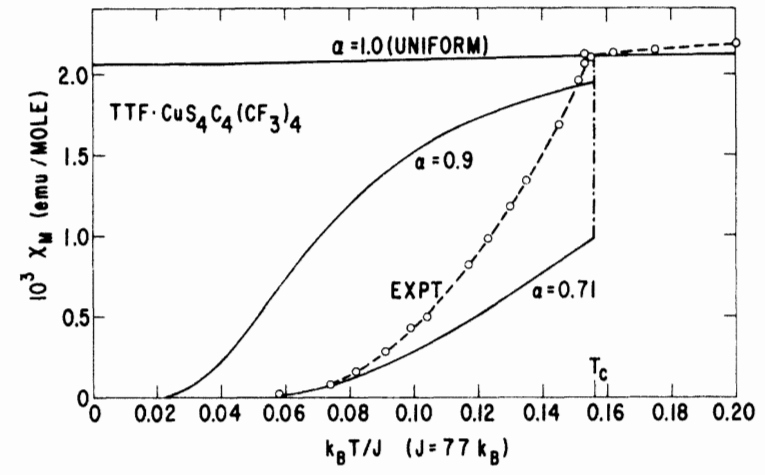

FIG. 14. Comparison between low-temperature experimental data for TTF $-\mathrm{CuS}_{4} \mathrm{C}_{4}\left(\mathrm{CF}_{3}\right)_{4}$ and calculated susceptibility for the AF chain with temperature-independent alternation, for various values of $\alpha$.

The static susceptibility data for Fig. 5 and the E PR results for Figs. 7 and 8 show that $\chi$ decreases sharply in all principal directions at $12 \mathrm{~K}$ for the $\mathrm{Cu}$ compound and $2.1 \mathrm{~K}$ for the $\mathrm{Au}$ compound. This behavior is incompatible with $3-\mathrm{D}$ or 2-D AF ordering of the spin systems, for which $\chi$ would decrease to zero for at most one orientation of the sample. Therefore we seek a model for which $\chi$ decreases to zero in all orientations. The simplest such model is the $1-\mathrm{D}$ Heisenberg chain with temperature-independent alternating exchange noted in Sec. II and shown in Fig. 1 for the weakly-alternating case $\alpha=0.95$. Our calculations for several large $\alpha$ values fail to reproduce the sharpness of the decrease in $\chi$ at $12 \mathrm{~K}$ ( $\mathrm{Cu}$ compound). This failure is further demonstrated in Fig. 14. While the alternation parameter value $\alpha=0.71$ fits the lowest temperature susceptibility, it falls short of the observed $\chi$ by a factor of 2 near the knee $\left(T_{c}\right)$. At the same time this figure does show that a progressive dimerization, i.e., temperature-dependent $\alpha$, could satisfactorily describe the observed susceptibility. As $\chi$ falls, there is some value $\alpha(T) \leqslant 1$ which will permit a fit at each temperature. As discussed in Sec. II, this feature is central to the spin-Peierls transition.

\section{B. Theory}

The basic Hamiltonian for the spin-Peierls system is

$$
\begin{aligned}
H= & \sum_{l} J(l, l+1)\left(\overrightarrow{\mathrm{S}}_{l} \cdot \overrightarrow{\mathrm{S}}_{l+1}\right) \\
& +\sum \omega_{0}(\overrightarrow{\mathrm{q}}, \alpha) b_{\overrightarrow{\mathrm{q}} \alpha}^{\dagger} b_{\overrightarrow{\mathrm{q}} \alpha},
\end{aligned}
$$

where the sum over lattice sites $l$ includes nearest intrachain neighbors only, $b_{\vec{a} \alpha}^{\dagger}\left(b_{\vec{q} \alpha}\right)$ is the creation (destruction) operator for $3-D$ phonons, 
with wave vector $\vec{q}$ on branch $\alpha$, and $\omega_{0}$ is the unrenormalized phonon energy. Since the exchange energy $J(l, l+1)$ is a function of the $3-\mathrm{D}$ spatial separation of sites $l$ and $l+1$, the $1-\mathrm{D}$ spin inter actions depend on the 3-D motion of the lattice sites. Thus we are treating a $1-D$ magnetic system coupled to a $3-\mathrm{D}$ phonon system. (We take $\hbar=k_{B}=1$.)

Pytte $^{23}$ has treated this Hamiltonian in mean field. He used the Jordan-Wigner transformation from spin to pseudofermion operators. It is his mean-field treatment of basically $1-D$ equations which leads to a finite spin-Peierls transition temperature $T_{c}$. The $3-\mathrm{D}$ phonons are used only implicitly to justify this result, which is

$$
T_{c}=2.28 p J e^{-1 / \lambda},
$$

where $p(T)$ is a very slowly varying function of $T$. [We may use $p(T)=p(0)=1.6366$ for $T \leqslant T_{c}$.] The spin-phonon coupling constant $\lambda$ is defined by

$$
\lambda \equiv 4 \hat{g}^{2} p / \omega_{0}^{2} \pi J,
$$

where

$$
\begin{aligned}
& \omega_{0} \equiv \omega_{0}\left(\alpha \overrightarrow{\mathrm{q}}, q=2 k_{F}\right), \\
& \hat{g} \equiv \hat{g}\left(\alpha \overrightarrow{\mathrm{q}}, q=2 k_{F}\right)=\overrightarrow{\mathrm{e}}(\alpha \overrightarrow{\mathrm{q}}) \cdot \vec{\nabla}_{l} J(l, l+1) /(m N)^{1 / 2} .
\end{aligned}
$$

$\overrightarrow{\mathrm{e}}(\alpha \overrightarrow{\mathrm{q}})$ is the phonon polarization vector for branch $\alpha$. Pytte's analysis and our extensions are used in Ref. 1 to fit the susceptibility data. Above $T_{c}$, the chains are uniform, and $\chi$ is fit by the Bonner-Fisher curve. Below $T$, the lattice progressively dimerizes, and two unequal and alternating $J$ 's are produced,

$$
J_{1,2}=J[1 \pm \delta(T)] \text {. }
$$

From Ref. 1,

$$
\delta(T)=\Delta(T) / p J,
$$

where $\Delta(T)$ is the magnetic gap, which follows the usual BCS temperature dependence. For $T_{c}=12 \mathrm{~K}$ and $J=77 \mathrm{~K}$ (the Cu compound), the model pre$\operatorname{dicts}^{1} \delta(0)=0.167$. We therefore have available a prediction of

$$
\frac{J_{2}(T)}{J_{1}(T)}=\frac{1-\delta(T)}{1+\delta(T)}=\alpha(T)
$$

for all $T \leqslant T_{c}=12 \mathrm{~K}$. Using the Bulaevskii ${ }^{18}$ model of a dimerized chain [see Eq. (1)], we then calculate $\chi(T)$ to compare with the data.

A remark concerning a possible lattice effect omitted thus far is appropriate at this point. This is concomitant chain expansion or contraction, which is considered by Beni and Pincus. ${ }^{24}$ The obvious consequence of this effect is a change of $J$ as a function of temperature. Such temperature dependence must be factored into $J_{1,2}(T)$ [Eq. (6)].
In Ref. 1, $J_{1}(T)$ was held fixed, which amounts to a concomitant lattice expansion, and agreement was obtained with the $\chi(T)$ data using the predicted mean-field parameters [i.e., $\delta(0)=0.167$ ]. We have now performed the calculation allowing $J_{1,2}(T)$ to vary exactly as in Eq. (6) (i.e., no lattice change) and find that a value of $\delta(0)=0.127$ is needed to fit $\chi(T)$ for $T \leqslant 12 \mathrm{~K}$. The fit is as good as in Ref. 1 (see Fig. 5 ) but $J_{1}(0) / J_{2}(0)$ is $\sim 8 \%$ different than the mean-field values predicted above. This also corresponds to a mean-field $T_{c}$ of about $9 \mathrm{~K}$ which is a deviation of about $25 \%$ from the observed value. These deviations could be due to either inadequacies of the mean-field theory, the actual presence of lattice expansion, or both. Until low-temperature $\mathrm{x}$-ray or neutron studies are done, lattice change will remain an unknown parameter.

For the Au compound, knowledge of $J$ and $T_{c}$ ( 68 and $2.1 \mathrm{~K}$, respectively) determines the meanfield parameters:

$$
\Delta(0)=3.7 \mathrm{~K} ; \quad \delta(0)=0.033 ; \lambda=0.23 \text {. }
$$

In the absence of EPR intensity (or susceptibility). results for the $\mathrm{Au}$ compound extending down to $T / T_{c} \lessgtr 0.5$, we do not try to fit the low-temperature regime. The general shape in Fig. 7 just below $T_{c}$ clearly reflects the behavior seen in detail for the $\mathrm{Cu}$ compound in Fig. 5.

The model allows us to make the same meanfield predictions for the Au compound as we did for the $\mathrm{Cu}$ one, ${ }^{1}$ keeping in mind their well-known limitations. The magnetic specific-heat jump at $T_{c}$ should be BCS-like and $\sim 0.02 R\left(0.02 k_{B}\right.$ per formula unit) (cf., $\sim 0.1 R$ for $\mathrm{Cu}$ ). If we assume that only the TTF's are important in the $2 k_{F}$ lattice motion, we may say that $\omega_{0}$ is the same for both compounds. Then from Eq. (5), $\hat{g}_{\mathrm{Au}} / \hat{g}_{\mathrm{Cu}}=0.8$. If we define generalized lattice distortions ${ }^{23}\langle Q\rangle$ $=J \delta(T) / 2 \hat{g}$, we get $\langle Q\rangle_{\mathrm{Au}} /\langle Q\rangle_{\mathrm{Cu}}=0.216$. For $\omega_{0}$ $\simeq 90 \mathrm{~K},\langle Q\rangle_{\mathrm{Au}} \sim 0.004$ at $T=0$, which translates roughly to a $0.06 \%$ translational distortion along the $c$ axis (cf., $0.3 \%$ for $\mathrm{Cu}$ ). Of course, the distortion may be torsional or librational as well as translational. As discussed by Pytte, large magnetic fields can have interesting effects. For instance, the theory predicts a trimerization at $H$ $=1.1 \mathrm{~J} / \mathrm{g} \mu_{B} \sim 510 \mathrm{kOe}$ for the Au compound, assuming that nonlinear field effects have not entered.

Since the spin-Peierls transition has a structural instability, the $2 k_{F}$ phonon mode along the chains should exhibit soft-mode behavior. Bray and $\mathrm{Chui}^{32}$ have predicted that a soft mode should be visible within a few degrees of $T_{c}$ in the dynamic structure factor $S(\overrightarrow{\mathrm{q}}, \omega)$ at $\overrightarrow{\mathrm{q}} \equiv\left(\overrightarrow{\mathrm{q}}_{\perp}, q_{\|}\right)=\left(\vec{\gamma}, 2 k_{F}\right)$, where $\vec{\gamma}$ gives the minimum value of $\omega_{0}\left(\overrightarrow{\mathrm{q}}_{\perp}, 2 k_{F}\right)$, probably $\vec{\gamma}=0=\overrightarrow{\mathrm{q}}_{\perp}$. In addition a central peak should 
be visible in $S(\overrightarrow{\mathrm{q}}, \omega=0)$ for all $\overrightarrow{\mathrm{q}}_{\perp}$ to temperature well above $T_{c}$ 。

Mean-field predictions of $T_{c}$ for the regular Peierls transition in other quasi-1-D systems (e.g., TTF-TCNQ) are typically almost an order of magnitude too high. ${ }^{33}$ In comparison, the experimental $T_{c}$ for these spin-Peierls systems is more consistent with mean-field theory. An ostensible reason is that the "scale" energies for the two transitions are different: $p J$ for spin-Peierls vs Fermi energy $\left(E_{F}\right)$ for regular Peierls in Eq. (4). But the deeper reason must depend on the 3-D element of the problem implied by coupling to the phonon field. ${ }^{25}$ Indeed renormalizationgroup arguments ${ }^{32}$ emphasize the role of the phonons in the character of the spin-Peierls transition. A related question is why the effects of the phonon field are not easily observed in the static susceptibility data for $T>T_{c}$. The reason ${ }^{32}$ may be that the $\chi$ data are taken at $q_{11}=0$, a value at which the spin-phonon coupling constants $(\hat{g})$ go to zero. ${ }^{23,32}$

We note that all of the theoretical approaches to a spin-Peierls transition have resulted in secondorder transitions. Pouget et $a l .^{34}$ have pointed out that strong coupling and lattice compressibility (after Bean and Rodbell ${ }^{35}$ ) might produce a firstorder transition. (We note that Dubois and Carton ${ }^{21}$ find a second-order transition for strong coupling with the $X Y$ model.) The existing approaches have restricted themselves to a linear term in the spatial expansion of $J$. As noted in Sec. II, the Isingmodel spin system gave different answers to the spin-Peierls problem when $J$ was permitted a nonlinear variation. ${ }^{21,25}$

In the historical review in Sec. II, culminating in the theoretical treatment outlined above, we have not made reference to an extensive and rapidly growing body of theoretical work on cooperative phenomena in magnetoelastic systems. Here the dimensionality of the spin system has generally been taken to be the same as that of the phonon system. It appears that the nature of the phase transition depends crucially on the original formulation of the problem; for example, how the phonon-phonon and spin-phonon coupling, the surface effects, and the elastic anisotropy are taken into account. Factors such as these are likewise expected to affect the nature of the critical order parameter. ${ }^{36}$ Clearly the theory of magnetoelastic critical phenomena has not yet reached maturity, and in any case, none of these more sophisticated approaches has so far been directly applicable to the system studied here.

\section{EPR linewidth}

Resonance studies (both NMR and EPR) are fruitful tools for examining the dynamics of lowdimensional spin systems. Although there have been several reviews, ${ }^{37,38}$ it seems safe to say that the present understanding of the temperature dependence of the EPR linewidth, $\Delta H$, in these systems is unsatisfactory. ${ }^{39}$

Our results in Figs. 11 and 12 span what can be called the intermediate temperature region, i.e., low enough for the effects of short-range order to enter in but too high for well-defined spin waves. [Note $J / k_{B} \sim T\left(\chi_{\max }\right) \sim 50 \mathrm{~K}$.] From the current wis$\mathrm{dom}^{38}$ one expects the linewidths of $1-\mathrm{D}$ (and 2-D) antifer romagnets to go through a minimum in this region. In particular, at high temperature, spin diffusion theory indicates that the long-wavelength $q \rightarrow 0$ modes dominate the relaxation process. This contribution is proportional to $\chi T,{ }^{39}$ which we have scaled from the present experiments to match $\Delta H$ just below the structural transition. At the other extreme, as the temperature is lowered, the $q=0$ modes fade in intensity in favor of staggered AF susceptibility modes at the zone boundary, whose intensity rises rapidly with decreasing temperature, causing the linewidth to broaden again.

While 2-D systems have been observed which fit this picture, ${ }^{37,38}$ it has been less frequently the case for 1-D materials. In the latter, such a good model system as $^{40}$ TMMC $\left[\left(\mathrm{CH}_{3}\right)_{4} \mathrm{NMnCl}_{3}\right]$ exhibits a $\Delta H$ which is at first constant and then rises markedly as the temperature decreases. Very recent results, however, on CPC $\left(\mathrm{CuCl}_{2} \cdot 2 \mathrm{NC}_{5} \mathrm{H}_{5}\right),{ }^{39}$ and earlier data on ${ }^{41}$ $\mathrm{Cu}\left(\mathrm{C}_{6} \mathrm{H}_{5} \mathrm{COO}\right)_{2} \cdot 3 \mathrm{H}_{2} \mathrm{O}$ do follow the expected behavior.

With this background we note that our result for $\mathrm{TTF} \cdot \mathrm{CuS}_{4} \mathrm{C}_{4}\left(\mathrm{CF}_{3}\right)_{4}$ shows a shallow minimum (40 to $60 \mathrm{~K}$ depending on orientation) and tends toward $\chi T$ at high temperature. After the experimental gap between 10 and $4 \mathrm{~K}$, totally different behavior below $4 \mathrm{~K}$ is observed. In contrast is the result for the Au compound, which follows $\chi T$ almost exactly from 200 down to $4 \mathrm{~K}$.

Below the spin-Peierls transition in the copper salt, a single broad line is observed at $4.2 \mathrm{~K}$ in the approximate position of the $\mathrm{TTF}^{+}$resonance. Its width is strongly angle dependent varying from maxima of $\sim 13.5$ Oe peak-to-peak derivative at $\overrightarrow{\mathrm{H}}\|\overrightarrow{\mathrm{b}}, \overrightarrow{\mathrm{H}}\| \overrightarrow{\mathrm{c}}$ to a minimum of $\sim 5.5$ Oe with $\overrightarrow{\mathrm{H}}$ approximately $50^{\circ}$ from the $b$ axis in the $b c$ plane. Upon lowering the temperature, the line first broadens, then narrows, revealing the presence of other spectra ( $B$ and $C$ in Table II as well as other weaker ones) in addition to that of the $\mathrm{TTF}^{+}$, see Fig. 11. All of the spectra narrow in the same temperature region suggesting a common mechanism for their linewidths.

This behavior suggests exchange averaging between the spin systems..$^{42,43}$ The effective ex change frequency, $\nu_{e}$, will be temperature depen- 
dent because it reflects the concentration of the $\mathrm{TTF}^{+}$spins which are freezing out. Above $T_{c}$ $=12 \mathrm{~K}$, the high concentration of $\mathrm{TTF}^{+}$spins as sures a strongly exchange-narrowed line, relatively unaffected by the presence of the low concentration of the $B$ and $C$ spins. Below $T_{c}$, as the $\mathrm{TTF}^{+}$spins condense out, the effective exchange frequency decreases, in proportion to the $\mathrm{TTF}^{+}$concentration. When the average exchange frequency becomes comparable to an "average" frequency separation $\left(\Delta g \mu_{B} H / h\right)$ between the $\mathrm{TTF}^{+}$ and the other spins, the linewidth should go through a maximum and, as the exchange frequency decreases further, the individual spectra should emerge with individual widths given approximately by ${ }^{42}$

$$
g \mu_{B}\left(\Delta H-\Delta H_{0}\right) \cong 3^{1 / 2} h \nu_{e}
$$

Here $\Delta H_{0}$ is the natural width of the line involved, in the absence of exchange. [The exact constant of proportionality in Eq. (9) depends upon the relative concentrations of the participating spin systems. ${ }^{43}$ The equality given here is derived for exchange between two spin systems of equal concentration.

In the freeze-out region, the concentration of $\mathrm{TTF}^{+}$spins should be proportional to $e^{-\Delta(\boldsymbol{T}) / T}$, where $\Delta(T)$ is the spin-Peierls gap. In mean field at $T \leqslant 4.2 \mathrm{~K}, \Delta(T) \sim \Delta(0)=21 \mathrm{~K}$. The model of exchange broadening therefore predicts for the linevidths below $4.2 \mathrm{~K}, \Delta H-\Delta H_{0} \sim e^{-21 / T}$.

In Fig. 15 we have plotted the linewidth for the $\mathrm{TTF}^{+} \mathrm{EPR}$ line as it emerges with decreasing temperature from the broad line at $4.2 \mathrm{~K}$. Also plotted are values for $\Delta H-\Delta H_{0}$, where $\Delta H_{0}$ was estimated to be $4.0 \mathrm{Oe}$, the limiting value at the lowest temperature. These points are not sufficiently accurate nor do they span a large enough range in $\Delta H$ to give an accurate estimate of $\nu_{e}$, Eq. (9). Instead, we show the curve $v_{e}=10^{10} e^{-21 / T}$, which represents a fit to the "expected" $e^{-21 / T}$ dependence. [ The fit is not very sensitive to the value used for $\Delta(0)$.] The experimental results are clearly consistent with this interpretation. (We note that accurate studies in the future over a wider range of temperatures, in the exchange nar rowing region $\geqslant 4.2 \mathrm{~K}$ and at lower temperatures with other techniques to measure $T_{2}$, might serve as a sensitive independent measurement of the Peierls gap and its temperature dependence.)

Further confirmation of the role of exchange in the low-temperature linewidths comes from the angular dependence of the widths at $4.2 \mathrm{~K}$. The maxima in the width for $\overrightarrow{\mathrm{H}}\|\overrightarrow{\mathrm{b}}, \overrightarrow{\mathrm{H}}\| \overrightarrow{\mathrm{c}}$ corresponds to the orientation where the $\mathrm{TTF}^{+}, B$ and $C$ spectra, which are being averaged, have their largest separation. The minimurn width corresponds to

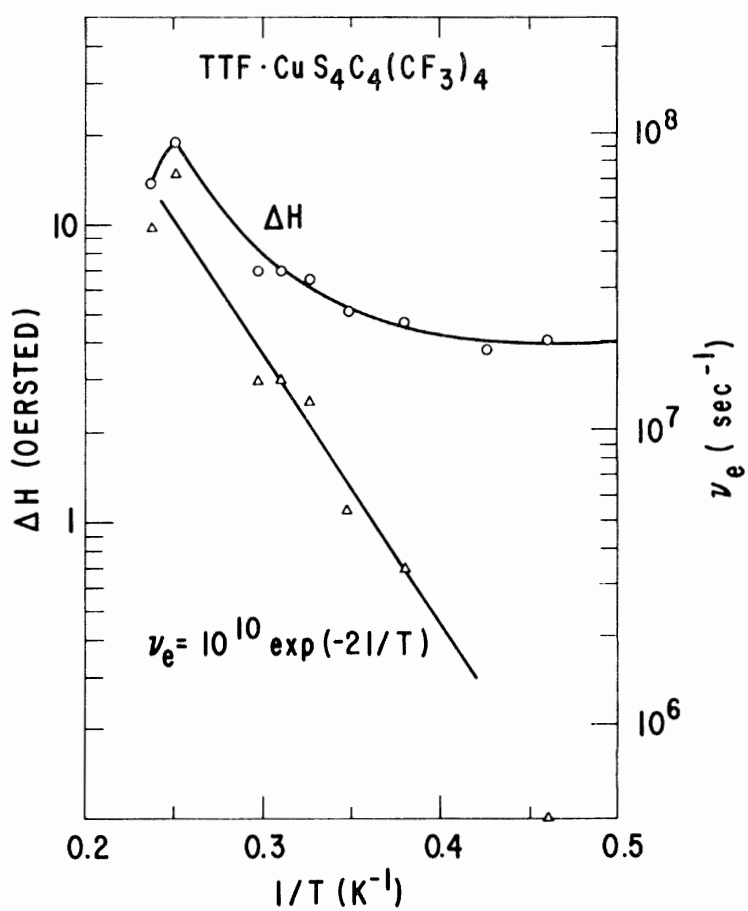

FIG. 15. EPR peak-to-peak derivative linewidth $\Delta H$ at $20 \mathrm{GHz}$ vs reciprocal temperature for $\mathrm{TTF}^{+}$in $\mathrm{TTF} \cdot \mathrm{CuS}_{4} \mathrm{C}_{4}\left(\mathrm{CF}_{3}\right)_{4}, \overrightarrow{\mathrm{H}} \| \overrightarrow{\mathrm{c}}$. Also shown is the inferred average exchange frequency $\nu_{e}$, matched to $(\Delta H-4.0)$ Oe (triangles), with the "expected" $e^{-21 / T}$ dependence.

the orientation where the $\mathrm{TTF}^{+}$and $B$ spectra superpose. Asymmetry in the angular dependence around this minimum can be explained by a lesser contribution from exchange with spectrum $C$. This dominance of the $\mathrm{TTF}^{+}-B$ exchange is consistent with the greater intensity of the $B$ spectrum vs that of $C(\sim 2: 1)$. It may also reflect lesser exchange with the $C$ spin system due to geometrical effects (sublattice position, etc.).

The $\mathrm{TTF}^{+}$spins excited across the spin-Peierls gap must be formed in pairs, and as such, are triplet excitons. Soos ${ }^{44}$ has summarized the EPR characteristics expected for triplet excitons: (i) For a Frenkel exciton, the two spins remain adjacent in the crystal and fine structure due to dipole-dipole interactions between the spins can be anticipated at low enough temperatures for exchange averaging to be unimportant. (ii) For a Wannier exciton, the spins move independently throughout the crystal and no fine structure is predicted. We observe no $S=1$ fine structure, a fact which implies Wannier excitons in our case. This is consistent with observations in other or-. ganic free radicals, Frenkel excitons being observed only in systems in which the alternation parameter, Eq. (6), $\delta \sim 1 .^{44}$ It is also consistent with observations ${ }^{45}$ that in the nonalternating 
Heisenberg AF limit the triplet excitations have a delocalized rather than a bound character, in contrast to the ferromagnetic limit where both types of states occur.

\section{COMPARISON WITH EARLIER SUGGESTED EXAMPLES}

We noted in Sec. I that various materials have been suggested as examples of what we now call spin-Peierls behavior. Earliest among these was Würster's blue perchlorate (N, $N^{\prime}$-tetramethyl- $p$ phenylenediamine perchlorate). More recently proposed materials are the alkali-TCNQ salts ${ }^{46}$ (e.g., Li tetracyanoquinodimethane) and chromiumdoped or uniaxially stressed $\mathrm{VO}_{2}$. We now examine their properties in the light of the model of the transition developed above.

Würster's blue perchlorate shows magnetic behavior which provided major stimuli to McConnell et $a l .^{8}$ and Chesnut $^{15}$ for the first qualitative and quantitative treatments, respectively, of the instability of a system of magnetic insulator chains against dimerization. This material has a firstorder transition ${ }^{47,48}$ at $190 \mathrm{~K}$. Its magnetic sus ceptibility has been analyzed ${ }^{49}$ as that of a regular Heisenberg AF chain $\left(J / k_{B}=200 \mathrm{~K}\right)$ above that transition and of a strongly dimerized chain ( $\delta$ $=0.75, J / k_{B}=190 \mathrm{~K}$ ) below. Both Chesnut and, later, Beni and Pincus ${ }^{24}$ having produced theories with second-order transitions, concluded that their models did not match this material. In Sec. IV B we remarked that while a model yielding a firstorder transition has not been demonstrated, such a possibility cannot be ruled out at this time. How ever, it is generally agreed that the first-order transition in Würster's blue perchlorate results from a crystal structure instability in which the magnetic system plays no important role. A detailed crystal-structure study ${ }^{50}$ shows that the $\mathrm{ClO}_{4}^{-}$groups are disordered (rotating) at room temperature and lose most of that disorder at low temperature. This compound is therefore not an example of a spin-Peierls material.

The alkali-TCNQ salts belong to the class of organic ion-radical crystals ${ }^{7}$ whose interesting electrical and magnetic properties have drawn much attention recently. These materials are semiconducting in contrast to the insulators we have been discussing. While susceptibility curves for some members of the series resemble the low-temperature part, i.e., for $T<T\left(\chi_{\max }\right)$, of our data, there remains considerable disagreement between groups of investigators..$^{51-53}$ This is particularly so for Li-TCNQ, which has received careful study. Each member of the series shows a transition in magnetic behavior at a temperature between $\sim 150$ and $400 \mathrm{~K}$ which is first order for some and per- haps second order for others in the series. ${ }^{47,54}$

The question of applying a Heisenberg model of magnetic behavior to a semiconductor depends on the relative magnitudes of the parameters of the Hubbard Hamiltonian: $U$, the on-site repulsive energy and $t$, the one-electron transfer energy. For the Heisenberg model one requires $t \ll U$, a condition met for these salts in the estimation of some investigator $\mathrm{s}^{46,51}$ and not met in the opinion of others..$^{52,53}$ At best, there is insufficient evidence for characteristic $1-D$ magnetic behavior above the transitions observed. Further, Khanna et $a l .{ }^{51}$ show an excellent fit to a temperature-independent magnetic (singlet-triplet) gap of $1300 \mathrm{~K}$ below the transition at $225 \mathrm{~K}$ in Li-TCNQ. That feature is also inconsistent with the spin-Peierls concept, apart from any question of the order of the transition. The large gap means that the density of magnetic excitations at $225 \mathrm{~K}$ is quite small $\left(\sim 10^{-3}\right)$. Thus they argue that the associated magnetic entropy is so small that the transition in all likelihood is not magnetic in origin but rather is driven by a lattice structure instability.

We remark that it is a possibility that the $2 k_{F}$ instability (i.e., lattice dimerization) may be a general characteristic of chains with any finite Hubbard parameters $(t, U)$. In this case, the regular Peierls transition and spin-Peierls transition would be $t \gg U$ and $t \ll U$ limits, respectively, of some "general Peierls transition." Perhaps one could then classify the alkali-TCNQ salts as "intermediate" examples of this general Peierls transition.

The third example is $\mathrm{VO}_{2}$ modified by stress $\mathrm{s}^{55}$ or doping. ${ }^{56-58}$ The unmodified compound undergoes a first-order metal-to-insulator transition at $343 \mathrm{~K}$, from the rutile structure to a monoclinic $\left(M_{1}\right)$ phase where all $\mathrm{V}$ atoms are equivalent and paired. The effect of doping or stress is to introduce another monoclinic insulating phase $\left(M_{2}\right)$ which is stable for a few tens of degrees, just below the metallic rutile phase. Pouget et $a l .^{34}$ interpret the $M_{2}$ structure as two sets of $\mathrm{V}$ atom chains, one of which consists of dimerized pairs while the other is believed to be a regularly spaced zig-zag chain of $\mathrm{V}$ ions with localized spins. Between the phases $M_{2}$ and $M_{1}$, a transitional phase $(T)$ occurs in which the zigzag regular chain gradually becomes a dimerized chain. The transition $M_{2} \rightarrow T$ has either a firstor second-order character moving toward the latter with increasing $\mathrm{Cr}$ doping or stress. Generally similar results are obtained by $\mathrm{Fe}$ doping. ${ }^{58}$

In describing these systems, the authors invoke temparature-dependent exchange during the progressive dimerization, which is one identifying feature of the spin-Peierls model. They suggest 
that the first-order transitions could be explained by an appropriate ${ }^{35}$ strong-coupling theory. These systems may indeed have spin-Peierls instabilities, although one must worry about crystal structure instabilities in these complicated enviornments. More conclusive evidence for 1-D magnetic behavior in the $M_{2}$ phase would be helpful. Alternatively, a rather different version of the phase diagram for impurity-doped $\mathrm{VO}_{2}$ has recently been proposed. ${ }^{59}$ The region interpreted as the translational $(T)$ phase is indicated therein to be a mixed-phase region to which the progressive dimerization would not apply. We might also point out that a progressive dimerization of chains ${ }^{60}$ appears to be a feature of the (rutile) metal-semiconductor transition (not first order ${ }^{61}$ ) in $\mathrm{NbO}_{2}$, occurring at $1083 \mathrm{~K}$. Its susceptibility behavior ${ }^{62}$ is superficially similar to that of $\mathrm{Li}-\mathrm{TCNQ}$ and of $\mathrm{VO}_{2}: \mathrm{Cr}$, although a spin-Peierls model would be inappropriate.

\section{CONCLUSION}

We have reviewed the spin-Peierls transition as a progressive spin-lattice dimerization occurring below a transition temperature in a system of onedimensional antifer romagnetic Heisenberg chains. Its historical origins have been examined with emphasis on the background areas of metal-nonmetal transitions and spin-insulator magnetic model systems. Our magnetic susceptibility and EPR measurements on the planar $\pi$-donor-acceptor compounds TTF $\cdot M \mathrm{~S}_{4} \mathrm{C}_{4}\left(\mathrm{CF}_{3}\right)_{4}, M=\mathrm{Cu}, \mathrm{Au}$, demonstrate directly the characteristic properties with a textbook-model simplicity. The behavior is in reasonably good agreement with a mean-field theory (after Pytte ${ }^{23}$ ) embracing (1-D) uniform Heisenberg AF chains coupled to a 3-D phonon field. The susceptibility of the progressively alternating chain is calculated in a Hartree-Fock approximation using the Bulaevskii model. EPR linewidths for intermediate- and low-temperature regions are presented and discussed. Areas for further experimental work are pointed out, with estimates made for several observables. We have also critically examined the behavior of several other materials suggested as examples of this transition.

A decade of theoretical investigation has developed our understanding of the spin-Peierls transition but more theoretical work is needed. Nonlinear lattice dependence of the exchange energy, lattice compressibility effects, and the strong-coupling regime should be interesting to study in connection with first-order transitions. The interaction of the 1-D spin chains and 3-D phonons has not been completely and properly examined within the framework of a rigorous magnetoelastic theory. A general theory linking the metallic Peierls transition with the spin-Peierls transition should be developed.

\section{ACKNOWLEDGMENTS}

The authors wish to thank S. -T. Chui, V. J. Emery, A. J. Heeger, J. B. Torrance, and W. P. Wolf for valuable discussions. We are grateful to M. Tinkham for his hospitality in allowing one of us (D.E.P.) to use the SQUID magnetometer. We thank M. J. Jefferies for providing the $\mathrm{Nb}-\mathrm{Ti}$ wire and R. L. Rhodenizer for help in the design of the solenoid used with the Faraday balance. It is a pleasure to acknowledge the valuable technical assistance of W. R. Giard, L. A. Gruenke, and S. H. Wee.
*Work supported in part by a grant from the U.S. AFOSR under Contract No. F-44620-71-C-0129, and (for J. C. B.) by the U. S. ERDA.

$\dagger$ Present address: Physics Dept., Lehigh University, Bethlehem, Pa. 18015.

\$Present address: Physics Dept., Univ. of Rhode Island, Kingston, R. I. 02881.

${ }^{1}$ J. W. Bray, H. R. Hart, Jr., L. V. Interrante, I. S. Jacobs, J. S. Kasper, G. D. Watkins, S. H. Wee, and J. C. Bonner, Phys. Rev. Lett. 35, 744 (1975).

${ }^{2}$ For a recent review, see, e.g., R. A. Bari, J. Solid State Chem. 12, 383 (1975).

${ }^{3}$ R. E. Peierls, Quantum Theory of Solids (Oxford U. P., London, 1955), p. 108.

${ }^{4}$ For a recent review, see, e.g., L. J. deJongh and A. R. Miedema, Adv. Phys. 23, 1 (1974).

${ }^{5}$ L. V. Interrante, K. W. Browall, H. R. Hart, Jr., I. S. Jacobs, G. D. Watkins, and S. H. Wee, J. Am. Chem. Soc. 97,889 (1975); J. S. Kasper, L. V. Interrante, and C. A. Secaur, ibid. 97, 890 (1975).
${ }^{6}$ I. S. Jacobs, L. V. Interrante, and H. R. Hart, Jr. AIP Conf. Proc. 24, 355 (1975).

${ }^{7}$ See, e.g., Z. G. Soos, Ann. Rev. Phys. Chem. 27, 121 (1974).

${ }^{8}$ H. M. McConnell and R. Lynden-Bell, J. Chem. Phys. 36,2393 (1962); D. D. Thomas, H. Keller, and H. M. $\overline{\mathrm{Mc} C}$ Connell, ibid. 39, 2321 (1963).

${ }^{9}$ H. Fröhlich, Proc. R. Soc. A 223, 296 (1954).

${ }^{10}$ C. G. Kuper, Proc. R. Soc. A 227, 214 (1955).

${ }^{11}$ Y. Ooshika, J. Phys. Soc. Jpn. 12 , 1238, 1246 (1957); 14, 747 (1959).

${ }^{12} \mathrm{H}$ C. Longuet-Higgins and L. Salem, Proc. R. Soc. A 251, 172 (1959).

${ }^{13}$ J. R. Platt, J. Chem. Phys. 25, 80 (1956).

${ }^{14}$ H. Kuhn, J. Chem. Phys. 17, 1198 (1949).

${ }^{15}$ D. B. Chesnut, J. Chem. Phys. 45, 4677 (1966).

${ }^{16} \mathrm{~J}$. C. Bonner and M. E. Fisher, Phys. Rev. 135, A640 (1964).

${ }^{17}$ L. N. Bulaevskii, Zh. Eksp. Teor. Fiz. 43, 968 (1962). [Sov. Phys.-JETP 16, 685 (1963)]. 
${ }^{18}$ L. N. Bulaevskii, Zh. Eksp. Teor. Fiz. 44, 1008 (1963) [Sov. Phys.-JETP 17, 684 (1963)]; Fiz. Tverd. Tela 11, 1132 (1969) [Sov. Phys.-Solid State 11, 921 (1969)]. ${ }^{19}$ W. Duffy, Jr. and K. P. Barr, Phys. Rev. 165,647 (1968).

${ }^{20}$ P. Pincus, Solid State Commun. 9, 1971 (1971).

${ }^{21}$ J. Y. Dubois and J. B. Carton, J. Phys. (Paris) 35, 371 (1974).

${ }^{22}$ G. Beni, J. Chem. Phys. 58, 3200 (1973).

${ }^{23}$ E. Pytte, Phys. Rev. B 10, 4637 (1974).

${ }^{24} \mathrm{G}$. Beni and P. Pincus, J. Chem. Phys. 57, 3531 (1972).

${ }^{25}$ E. Pytte, Phys. Rev. B 10, 2039 (1974).

${ }^{26}$ A. Luther and I. Pesche1, Phys. Rev. B 12, 3908 (1975).

${ }^{27}$ L. Alacer and A. H. Maki, J. Phys. Chem. 78, 215 (1974); W. E. Geiger, Jr., ibid. 77, 1862 (1973); J. Burgess, K. M. C. Davis, R. D. W. Kemmitt, J. B. Raynor, and J. Stocks, Inorg. Chim. Acta 4, 129 (1970); R. D. Schmitt, R. M. Wing, and A. H. Maki, J. Am. Chem. Soc. 91, 4394 (1969).

${ }^{28} \mathrm{~J}$. P. Gollub, M. R. Beasley, R. Callarotti, and M. Tinkham, Phys. Rev. B 7, 3039 (1973).

${ }^{29}$ G. D. Watkins and F. S. Ham. Phys. Rev. B 1,4071 (1970).

${ }^{30}$ R. H. Silsbee, Cruft Laboratory Technical Report No. 221 (Harvard University, 1956) (unpublished).

${ }^{31} \mathrm{~K}$. Nagata and Y. Tazuke, J. Phys. Soc. Jpn. $\underline{32}, 337$ (1972).

${ }^{32}$ J. W. Bray and S.- T. Chui, Ferroelectrics (to be published).

${ }^{33}$ P. A. Lee, T. M. Rice, and P. W. Anderson, Phys. Rev. Lett. 31, 462 (1973).

${ }^{34}$ J. P. Pouget, H. Launois, T. M. Rice, P. Dernier, A. Gossard, G. Villeneuve, and P. Hagenmuller, Phys. Rev. B 10, 1801 (1974).

${ }^{35}$ C. P. Bean and D. S. Rodbell, Phys. Rev. 126, 104 (1962).

${ }^{36}$ D. J. Bergman and B. I. Halperin, Phys. Rev. B 13 , 2145 (1976); M. A. deMoura, T. C. Lubensky, Y. Imry, and A. Aharony, Phys. Rev. B 13, 2176 (1976).

${ }^{37}$ D. Hone, AIP Conf. Proc. $\underline{5}, 4 \overline{13}$ (1972).

${ }^{38} \mathrm{D}$. W. Hone and P. M. Richards, Ann. Rev. Mat. Sci. 4, 337 (1974).

${ }^{39} \bar{Y}$. Ajiro, S. Matsukawa, T. Yamada, and T. Haseda, J. Phys. Soc. Jpn. 39, 259 (1975).
${ }^{40}$ R. E. Dietz, quoted in Ref. 37.

${ }^{41}$ K. Okuda, H. Hata, and M. Date, J. Phys. Soc. Jpn. 33,1574 (1972).

${ }^{42} \bar{M}$. T. Jones and D. B. Chesnut, J. Chem. Phys. 38 , 1311 (1963).

${ }^{43}$ H. S. Gutowsky and A. Saika, J. Chem. Phys. 25, 1228 (1956).

${ }^{44}$ Z. G. Soos, J. Chem. Phys. 46, 4284 (1967).

${ }^{45} \mathrm{~J}$. C. Bonner, B. Sutherland, and P. M. Richards, AIP Conf. Proc. 24, 335 (1975).

${ }^{46} \mathrm{~J}$. B. Torrance (personal communication).

${ }^{47} \mathrm{H}$. Terauchi, N. Sakamoto, and I. Shirotani, J. Chem. Phys. 64, 437 (1976).

${ }^{48} \mathrm{H}$. Chihara, M. Nakamura, and S. Seki, Bull. Chem. Soc. Jpn. 38, 1776 (1965).

${ }^{49}$ Z. G. Soos and R. G. Hughes, J. Chem. Phys. 46, 253 (1967).

${ }^{50}$ J. L. DeBoer and A. Vos, Acta Crystallogr. B 28, 835 (1972).

${ }^{51}$ S. K. Khanna, E. Ehrenfreund, E. F. Rybaczewski, and S. Etemad, AIP Conf. Proc. 10, 1509 (1973).

${ }^{52}$ J. G. Vegter, J. Kommandeur, and P. A. Fedders, Phys. Rev. B 7, 2929 (1973).

${ }^{53} \mathrm{~J}$. Kommandeur in Low-Dimensional Cooperative Phenomena, edited by H. J. Keller (Plenum, New York, 1975), p. 65.

${ }^{54} \mathrm{~J}$. G. Vegter, thesis (University of Groningen, 1972) (unpublished).

${ }^{55}$ J. P. Pouget, H. Launois, J. P. D'Haenens, P. Merenda, and T. M. Rice, Phys. Rev. Lett. 35, 873 (1975).

${ }^{56}$ J. P. D'Haenens, D. Kaplan, and P. Merenda, J. Phys. C 8, 2267 (1975).

${ }^{57}$ G. Villeneuve, M. Drillon, J. C. Launay, E. Marquestaut, and P. Hagenmuller, Solid State Commun. 17, 657 (1975).

${ }^{58}$ E. Pollert, G. Villeneuve, F. Ménil, and P. Hagenmuller, Mat. Res. Bull. 11, 159 (1976).

${ }^{59}$ J. M. Reyes, S. L. Segel, and M. Sayer, Can. J. Phys. 54,1 (1976).

${ }^{60}$ R. Pynn, J. D. Axe, and R. Thomas, Phys. Rev. B $\underline{13}$, 2965 (1976).

${ }^{61}$ R. Pynn and J. D. Axe, J. Phys. C 9 , L199 (1976).

${ }^{62}$ K. Sakata, J. Phys. Soc. Jpn. 26, 867 (1969). 